\title{
On more general boundary value problems involving sequential fractional derivatives
}

\author{
Bashir Ahmad ${ }^{1}$, Ahmed Alsaedi ${ }^{*}$ (D), Sotiris K. Ntouyas ${ }^{1,2}$ and Wafa Shammakh
}

"Correspondence: aalsaedi@hotmail.com

${ }^{1}$ Nonlinear Analysis and Applied Mathematics (NAAM)-Research

Group, Department of Mathematics, Faculty of Science, King Abdulaziz

University, Jeddah, Saudi Arabia Full list of author information is available at the end of the article

\begin{abstract}
We investigate the existence of solutions for new boundary value problems of Caputo-type sequential fractional differential equations and inclusions supplemented with nonlocal integro-multipoint boundary conditions. We apply the modern techniques of functional analysis to obtain the main results. We emphasize that the results presented in this paper are new and specialize to some known theorems with an appropriate choice of the parameters involved in the problems at hand.
\end{abstract}

MSC: 34A08; 34B10; 34B15; 34A60

Keywords: Sequential fractional derivative; Inclusions; Nonlocal; Integro-multipoint boundary conditions; Existence

\section{Introduction}

In this paper, we investigate the existence criteria for the solutions of Caputo-type sequential fractional differential equations and inclusions:

$$
\begin{array}{ll}
\left({ }^{c} D^{q}+\mu^{c} D^{q-1}\right) x(t)=f\left(t, x(t),{ }^{c} D^{\kappa} x(t)\right), & t \in[0,1], \\
\left({ }^{c} D^{q}+\mu^{c} D^{q-1}\right) x(t) \in F\left(t, x(t),{ }^{c} D^{\kappa} x(t)\right), & t \in[0,1],
\end{array}
$$

supplemented with nonlocal integro-multipoint boundary conditions:

$$
\left\{\begin{array}{l}
\rho_{1} x(0)+\rho_{2} x(1)=\sum_{i=1}^{m-2} \alpha_{i} x\left(\sigma_{i}\right)+\sum_{j=1}^{p-2} r_{j} \int_{\xi_{j}}^{\eta_{j}} x(s) d s, \\
\rho_{3} x^{\prime}(0)+\rho_{4} x^{\prime}(1)=\sum_{i=1}^{m-2} \delta_{i} x^{\prime}\left(\sigma_{i}\right)+\sum_{j=1}^{p-2} \gamma_{j} \int_{\xi_{j}}^{\eta_{j}} x^{\prime}(s) d s, \\
0<\sigma_{1}<\sigma_{2}<\cdots<\sigma_{m-2}<\cdots<\xi_{1}<\eta_{1}<\xi_{2}<\eta_{2}<\cdots<\xi_{p-2}<\eta_{p-2}<1,
\end{array}\right.
$$

where ${ }^{c} D^{q},{ }^{c} D^{\kappa}$ denote the Caputo fractional derivatives of order $q \in(1,2]$ and $\kappa \in(0,1)$, respectively, $\mu>0, f$ is a given continuous function, $F:[0,1] \times \mathbb{R} \times \mathbb{R} \rightarrow \mathcal{P}(\mathbb{R})$ is a multivalued map $(\mathcal{P}(\mathbb{R})$ is the family of all nonempty subsets of $\mathbb{R}), \rho_{1}, \rho_{2}, \rho_{3}, \rho_{4}$ are real constants and $\alpha_{i}, \delta_{i}(i=1,2, \ldots, m-2), r_{j}, \gamma_{j}(j=1,2, \ldots, p-2)$ are positive real constants.

The tools of fractional calculus such as fractional order differential and integral operators are found to be of great utility in developing the mathematical models related to dynamical systems involving fractals and chaos. The modelers' interest in such tools is due to the fact that fractional order operators are nonlocal in nature and can trace the

(c) The Author(s) 2019. This article is distributed under the terms of the Creative Commons Attribution 4.0 International License (http://creativecommons.org/licenses/by/4.0/), which permits unrestricted use, distribution, and reproduction in any medium, provided you give appropriate credit to the original author(s) and the source, provide a link to the Creative Commons license, and indicate if changes were made. 
hereditary characteristics of many materials and processes involved in the problem. For a detailed account of the subject, for example, see the books [1-6] and papers [7-10].

Fractional order single- and multivalued boundary value problems involving different kinds of boundary conditions attracted significant attention during the last two decades. The literature on this topic is now much enriched and contains a variety of results ranging from the existence theory to the methods of solution for such problems [11-28].

The present work is motivated by a recent article [29] dealing with a fractional differential equation: ${ }^{c} D^{q} x(t)=f\left(t, x(t),{ }^{c} D^{\beta} x(t)\right), 1<q \leq 2,0<\beta<1, t \in(0,1)$, equipped with boundary conditions (1.3).

The rest of the paper is arranged as follows. In Sect. 2, we outline the basic concepts of fractional calculus and prove an auxiliary lemma. Section 3 contains the main results for the problem (1.1) and (1.3) and illustrative examples for the obtained results. In Sect. 4, we prove the existence of solutions for the inclusion problem (1.2) and (1.3) for convex- as well as nonconvex-valued maps involved in the given problem. The paper concludes with some interesting observations.

\section{Preliminaries}

Let us begin this section with some preliminary concepts of fractional calculus $[1,4]$.

Definition 2.1 Let $v$ be a locally integrable real-valued function on $-\infty \leq a<t<b \leq+\infty$. The Riemann-Liouville fractional integral $I_{a}^{\alpha}$ of $v$ of order $\alpha \in \mathbb{R}(\alpha>0)$ is defined as

$$
I_{a}^{\alpha} v(t)=\frac{1}{\Gamma(\alpha)} \int_{a}^{t}(t-s)^{\alpha-1} v(s) d s
$$

where $\Gamma$ denotes the Euler gamma function.

Definition 2.2 Let $v, v^{(m)} \in L^{1}[a, b]$ for $-\infty \leq a<t<b \leq+\infty$. The Riemann-Liouville fractional derivative $D_{a}^{\alpha}$ of $v$ of order $\alpha>0(m-1<\alpha<m, m \in \mathbb{N})$ is defined as

$$
D_{a}^{\alpha} \nu(t)=\frac{d^{m}}{d t^{m}} I_{a}^{m-\alpha} v(t)=\frac{1}{\Gamma(m-\alpha)} \frac{d^{m}}{d t^{m}} \int_{a}^{t}(t-s)^{m-1-\alpha} v(s) d s,
$$

while the Caputo fractional derivative ${ }^{c} D_{a}^{\alpha}$ of $v$ of order $\alpha \in \mathbb{R}(m-1<\alpha<m, m \in \mathbb{N})$ is defined as

$$
{ }^{c} D_{a}^{\alpha} v(t)=D_{a}^{\alpha}\left[v(t)-v(a)-v^{\prime}(a) \frac{(t-a)}{1 !}-\cdots-v^{(m-1)}(a) \frac{(t-a)^{m-1}}{(m-1) !}\right] .
$$

Remark 2.3 If $v \in C^{m}[a, b]$, then the Caputo fractional derivative ${ }^{c} D_{a}^{\alpha}$ of order $\alpha \in \mathbb{R}$ (m$1<\alpha<m, m \in \mathbb{N}$ ) is defined as

$$
{ }^{c} D_{a}^{\alpha} \nu(t)=I_{a}^{m-\alpha} v^{(m)}(t)=\frac{1}{\Gamma(m-\alpha)} \int_{a}^{t}(t-s)^{m-1-\alpha} v^{(m)}(s) d s .
$$

In the present work, we denote the Riemann-Liouville fractional integral $I_{a}^{\alpha}$ and the Caputo fractional derivative ${ }^{c} D_{a}^{\alpha}$ with $a=0$ by $I^{\alpha}$ and ${ }^{c} D^{\alpha}$, respectively.

To define the solution for problem (1.1) and (1.2), we consider the following lemma dealing with its linear variant. 
Lemma 2.4 Let $h \in C([0,1], \mathbb{R})$. Then the integral solution for the sequential fractional differential equation

$$
\left({ }^{c} D^{q}+\mu^{c} D^{q-1}\right) x(t)=h(t)
$$

supplemented with the boundary conditions (1.3) is given by

$$
\begin{aligned}
x(t)= & \sum_{i=1}^{m-2} \phi_{i}(t) \int_{0}^{\sigma_{i}} e^{\mu\left(s-\sigma_{i}\right)}\left(I^{q-1} h(s)\right) d s \\
& +\sum_{j=1}^{p-2} \psi_{j}(t) \int_{\xi_{j}}^{\eta_{j}}\left(\int_{0}^{s} e^{\mu(m-s)}\left(I^{q-1} h(m)\right) d m\right) d s \\
& +\Omega_{1}(t)\left[\sum_{i=1}^{m-2} \delta_{i} I^{q-1} h\left(\sigma_{i}\right)+\sum_{j=1}^{p-2} \gamma_{j} \int_{\xi_{j}}^{\eta_{j}}\left(I^{q-1} h(s)\right) d s-\rho_{4} I^{q-1} h(1)\right] \\
& +\Omega_{2}(t) \int_{0}^{1} e^{\mu(s-1)}\left(I^{q-1} h(s)\right) d s+\int_{0}^{t} e^{\mu(s-t)}\left(I^{q-1} h(s)\right) d s,
\end{aligned}
$$

where

$$
\begin{aligned}
& \phi_{i}(t)=\frac{1}{\mu \Delta}\left[\mu e^{-\mu t}\left(B_{2} \alpha_{i}+\mu B_{1} \delta_{i}\right)-\left(1-e^{-\mu t}\right)\left(A_{2} \alpha_{i}+\mu A_{1} \delta_{i}\right)\right], \\
& \psi_{j}(t)=\frac{1}{\mu \Delta}\left[\mu e^{-\mu t}\left(B_{2} r_{j}+\mu B_{1} \gamma_{j}\right)-\left(1-e^{-\mu t}\right)\left(A_{2} r_{j}+\mu A_{1} \gamma_{j}\right)\right], \\
& \Omega_{1}(t)=\frac{1}{-\mu \Delta}\left[\mu B_{1} e^{-\mu t}-A_{1}\left(1-e^{-\mu t}\right)\right], \\
& \Omega_{2}(t)=\frac{1}{-\mu \Delta}\left[\mu e^{-\mu t}\left(\rho_{4} \mu B_{1}+\rho_{2} B_{2}\right)-\left(1-e^{-\mu t}\right)\left(\rho_{4} \mu A_{1}+\rho_{2} A_{2}\right)\right], \\
& A_{1}=\rho_{1}+\rho_{2} e^{-\mu}-\sum_{i=1}^{m-2} \alpha_{i} e^{-\mu \sigma_{i}}+\sum_{j=1}^{p-2} \frac{r_{j}}{\mu}\left(e^{-\mu \eta_{j}}-e^{-\mu \xi_{j}}\right), \\
& A_{2}=-\mu\left[\rho_{3}+\rho_{4} e^{-\mu}-\sum_{i=1}^{m-2} \delta_{i} e^{-\mu \sigma_{i}}+\frac{1}{\mu} \sum_{j=1}^{p-2} \gamma_{j}\left(e^{-\mu \eta_{j}}-e^{-\mu \xi_{j}}\right)\right], \\
& B_{1}=\frac{1}{\mu}\left\{\rho_{2}\left(1-e^{-\mu}\right)-\sum_{i=1}^{m-2} \alpha_{i}\left(1-e^{-\mu \sigma_{i}}\right)-\sum_{j=1}^{p-2}\left[r_{j}\left(\eta_{j}-\xi_{j}\right)+\frac{1}{\mu}\left(e^{-\mu \eta_{j}}-e^{-\mu \xi_{j}}\right)\right]\right\}, \\
& B_{2}=\rho_{3}+\rho_{4} e^{-\mu}-\sum_{i=1}^{m-2} \delta_{i} e^{-\mu \sigma_{i}}+\frac{1}{\mu} \sum_{j=1}^{p-2}\left(e^{-\mu \eta_{j}}-e^{-\mu \xi_{j}}\right),
\end{aligned}
$$

with the assumption that

$$
\Delta=A_{1} B_{2}-B_{1} A_{2} \neq 0 .
$$

Proof Applying the integral operator $I^{q-1}$ on both sides of (2.1) and then solving the resulting equation, we get

$$
x(t)=\omega_{0} e^{-\mu t}+\frac{\omega_{1}}{\mu}\left(1-e^{-\mu t}\right)+\int_{0}^{t} e^{\mu(s-t)}\left(\int_{0}^{s} \frac{(s-\tau)^{q-2}}{\Gamma(q-1)} h(\tau) d \tau\right) d s,
$$


where $\omega_{i}(i=0,1)$ are unknown arbitrary constants. From (2.8), we have

$$
\begin{aligned}
x^{\prime}(t)= & -\mu \omega_{0} e^{-\mu t}+\omega_{1} e^{-\mu t}-\mu \int_{0}^{t} e^{\mu(s-t)}\left(\int_{0}^{s} \frac{(s-\tau)^{q-2}}{\Gamma(q-1)} h(\tau) d \tau\right) d s \\
& +\int_{0}^{t} \frac{(t-\tau)^{q-2}}{\Gamma(q-1)} h(\tau) d \tau .
\end{aligned}
$$

Using (2.8) and (2.9) in the boundary conditions (1.2), we obtain

$$
A_{1} \omega_{0}+B_{1} \omega_{1}=J_{1}, \quad A_{2} \omega_{0}+B_{2} \omega_{1}=J_{2},
$$

where $A_{i}$ and $B_{i}(i=1,2)$ are respectively given by (2.5) and (2.6), and

$$
\begin{aligned}
J_{1}= & \sum_{i=1}^{m-2} \alpha_{i} \int_{0}^{\sigma_{i}} e^{\mu\left(s-\sigma_{i}\right)}\left(I^{q-1} h(s)\right) d s+\sum_{j=1}^{p-2} r_{j} \int_{\xi_{j}}^{\eta_{j}}\left(\int_{0}^{s} e^{\mu(m-s)}\left(I^{q-1} h(m)\right) d m\right) d s \\
& -\rho_{2} \int_{0}^{1} e^{\mu(s-1)}\left(I^{q-1} h(s)\right) d s, \\
J_{2}= & \sum_{i=1}^{m-2} \delta_{i}\left[I^{q-1} h\left(\sigma_{i}\right)-\mu \int_{0}^{\sigma_{i}} e^{\mu\left(s-\sigma_{i}\right)}\left(I^{q-1} h(s)\right) d s\right] \\
& +\sum_{j=1}^{p-2} \gamma_{j}\left[\int_{\xi_{j}}^{\eta_{j}}\left(I^{q-1} h(s)\right) d s-\mu \int_{\xi_{j}}^{\eta_{j}}\left(\int_{0}^{s} e^{\mu(m-s)}\left(I^{q-1} h(m)\right) d m\right) d s\right] \\
& +\rho_{4}\left[\mu \int_{0}^{1} e^{\mu(s-1)}\left(I^{q-1} h(s)\right) d s-I^{q-1} h(1)\right] .
\end{aligned}
$$

Solving system (2.10) for the unknown constants $\omega_{0}, \omega_{1}$ yields

$$
\begin{aligned}
\omega_{0}= & \frac{1}{\Delta}\left\{\sum_{i=1}^{m-2}\left(B_{2} \alpha_{i}+\mu B_{1} \delta_{i}\right) \int_{0}^{\sigma_{i}} e^{\mu\left(s-\sigma_{i}\right)}\left(I^{q-1} h(s)\right) d s\right. \\
& +\sum_{j=1}^{p-2}\left(B_{2} r_{j}+\mu B_{1} \gamma_{j}\right) \int_{\xi_{j}}^{\eta_{j}}\left(\int_{0}^{s} e^{\mu(m-s)}\left(I^{q-1} h(m)\right) d m\right) d s \\
& -\left(\rho_{4} \mu B_{1}+\rho_{2} B_{2}\right) \int_{0}^{1}\left(e^{\mu(s-1)} I^{q-1} h(s)\right) d s \\
& \left.-B_{1} \sum_{i=1}^{m-2} \delta_{i} I^{q-1} h\left(\sigma_{i}\right)-B_{1} \sum_{j=1}^{p-2} \gamma_{j} \int_{\xi_{j}}^{\eta_{j}}\left(I^{q-1} h(s)\right) d s-\rho_{4} B_{1} I^{q-1} h(1)\right\}, \\
\omega_{1}= & \frac{1}{\Delta}\left\{-\sum_{i=1}^{m-2}\left(A_{2} \alpha_{i}+\mu A_{1} \delta_{i}\right) \int_{0}^{\sigma_{i}} e^{\mu\left(s-\sigma_{i}\right)}\left(I^{q-1} h(s)\right) d s\right. \\
& -\sum_{j=1}^{p-2}\left(A_{2} r_{j}+\mu A_{1} \gamma_{j}\right) \int_{\xi_{j}}^{\eta_{j}}\left(\int_{0}^{s} e^{\mu(m-s)}\left(I^{q-1} h(m)\right) d m\right) d s \\
& +A_{1} \sum_{i=1}^{m-2} \delta_{i} I^{q-1} h\left(\sigma_{i}\right)+A_{1} \sum_{j=1}^{p-2} \gamma_{j} \int_{\xi_{j}}^{\eta_{j}}\left(I^{q-1} h(s)\right) d s-\rho_{4} A_{1} I^{q-1} h(1) \\
& \left.+\left(\rho_{4} \mu A_{1}+\rho_{2} A_{2}\right) \int_{0}^{1}\left(e^{\mu(s-1)} I^{q-1} h(s)\right) d s\right\},
\end{aligned}
$$


where $\Delta$ is given by (2.7). Substituting the values of $\omega_{0}$ and $\omega_{1}$ into (2.8), together with the notations (2.3) and (2.4), we get the solution (2.2). The converse follows by direct computation. This completes the proof.

\section{Main results for the problem (1.1) and (1.3)}

Let $X=\left\{x \mid x \in C([0,1], \mathbb{R})\right.$ and $\left.{ }^{c} D^{\kappa} x \in C([0,1], \mathbb{R})\right\}$ be a space equipped with the norm $\|x\|_{X}=\sup _{t \in[0,1]}|x(t)|+\sup _{t \in[0,1]}\left|{ }^{c} D^{\kappa} x(t)\right|=\|x\|+\left\|{ }^{c} D^{\kappa} x\right\|$, where ${ }^{c} D^{\kappa}$ denotes the standard Caputo fractional derivative of order $0<\kappa \leq 1$. As argued in [30], $\left(X,\|\cdot\|_{X}\right)$ is a Banach space.

By means of Lemma 2.4, we transform problem (1.1) and (1.3) into a fixed point problem as $x=\mathfrak{H} x$, where the operator $\mathfrak{H}: X \rightarrow X$ is defined by

$$
\begin{aligned}
(\mathfrak{H} x)(t)= & \sum_{i=1}^{m-2} \phi_{i}(t) \int_{0}^{\sigma_{i}} e^{\mu\left(s-\sigma_{i}\right)}\left(I^{q-1} f\left(s, x(s),{ }^{c} D^{\kappa} x(s)\right)\right) d s \\
& +\sum_{j=1}^{p-2} \psi_{j}(t) \int_{\xi_{j}}^{\eta_{j}}\left(\int_{0}^{s} e^{\mu(m-s)}\left(I^{q-1} f\left(m, x(m),{ }^{c} D^{\kappa} x(m)\right)\right) d m\right) d s \\
& +\Omega_{1}(t)\left[\sum_{i=1}^{m-2} \delta_{i}\left(I^{q-1} f\left(\sigma_{i}, x\left(\sigma_{i}\right),{ }^{c} D^{\kappa} x\left(\sigma_{i}\right)\right)\right)\right. \\
& \left.+\sum_{j=1}^{p-2} \gamma_{j} \int_{\xi_{j}}^{\eta_{j}}\left(I^{q-1} f\left(s, x(s),{ }^{c} D^{\kappa} x(s)\right)\right) d s-\rho_{4} I^{q-1} f\left(1, x(1),{ }^{c} D^{\kappa} x(1)\right)\right] \\
& +\Omega_{2}(t) \int_{0}^{1} e^{\mu(s-1)}\left(I^{q-1} f\left(s, x(s),{ }^{c} D^{\kappa} x(s)\right)\right) d s \\
& +\int_{0}^{t} e^{\mu(s-t)}\left(I^{q-1} f\left(s, x(s),{ }^{c} D^{\kappa} x(s)\right)\right) d s, \quad t \in[0,1],
\end{aligned}
$$

where $\phi_{i}, \psi_{j}$ and $\Omega_{i}(i=1,2)$ are respectively given by (2.3) and (2.4). Furthermore, we have

$$
\begin{aligned}
(\mathfrak{H} x)^{\prime}(t)= & \sum_{i=1}^{m-2} \phi_{i}^{\prime}(t) \int_{0}^{\sigma_{i}} e^{\mu\left(s-\sigma_{i}\right)}\left(I^{q-1} f\left(s, x(s),{ }^{c} D^{\kappa} x(s)\right)\right) d s \\
& +\sum_{j=1}^{p-2} \psi_{j}^{\prime}(t) \int_{\xi_{j}}^{\eta_{j}}\left(\int_{0}^{s} e^{\mu(m-s)}\left(I^{q-1} f\left(m, x(m),{ }^{c} D^{\kappa} x(m)\right)\right) d m\right) d s \\
& +\Omega_{1}^{\prime}(t)\left[\sum_{i=1}^{m-2} \delta_{i}\left(I^{q-1} f\left(\sigma_{i}, x\left(\sigma_{i}\right),{ }^{c} D^{\kappa} x\left(\sigma_{i}\right)\right)\right)\right. \\
& \left.+\sum_{j=1}^{p-2} \gamma_{j} \int_{\xi_{j}}^{\eta_{j}}\left(I^{q-1} f\left(s, x(s),{ }^{c} D^{\kappa} x(s)\right)\right) d s-\rho_{4} I^{q-1} f\left(1, x(1),{ }^{c} D^{\kappa} x(1)\right)\right] \\
& +\Omega_{2}^{\prime}(t) \int_{0}^{1} e^{\mu(s-1)}\left(I^{q-1} f\left(s, x(s),{ }^{c} D^{\kappa} x(s)\right)\right) d s \\
& -\mu \int_{0}^{t} e^{\mu(s-t)}\left(I^{q-1} f\left(s, x(s),{ }^{c} D^{\kappa} x(s)\right)\right) d s \\
& +I^{q-1} f\left(t, x(t),{ }^{c} D^{\kappa} x(t)\right), \quad t \in[0,1],
\end{aligned}
$$


where

$$
\begin{aligned}
& \phi_{i}^{\prime}(t)=\frac{-e^{-\mu t}}{\Delta}\left[\mu\left(B_{2} \alpha_{i}+\mu B_{1} \delta_{i}\right)+A_{2} \alpha_{i}+\mu A_{1} \delta_{i}\right], \\
& \psi_{i}^{\prime}(t)=\frac{-e^{-\mu t}}{\Delta}\left[\mu\left(B_{2} r_{j}+\mu B_{1} \gamma_{j}\right)+A_{2} r_{j}+\mu A_{1} \gamma_{j}\right], \\
& \Omega_{1}^{\prime}(t)=\frac{e^{-\mu t}}{\Delta}\left[\mu B_{1}+A_{1}\right], \\
& \Omega_{2}^{\prime}(t)=\frac{e^{-\mu t}}{\Delta}\left[\mu\left(\rho_{4} \mu B_{1}+\rho_{2} B_{1}\right)+\rho_{4} \mu A_{1}+\rho_{2} A_{2}\right] .
\end{aligned}
$$

Next we fix some quantities as follows:

$$
\begin{aligned}
& \lambda=\frac{1}{\mu \Gamma(q)} \sum_{i=1}^{m-2} \widehat{\phi}_{i} \sigma_{i}^{q-1}\left(1-e^{-\mu \sigma_{i}}\right)+\frac{1}{\mu \Gamma(q+1)} \sum_{j=1}^{p-2} \widehat{\psi}_{j}\left(\eta_{j}^{q}-\xi_{j}^{q}\right)\left(1-e^{-\mu \eta_{j}}\right) \\
& +\frac{\widehat{\Omega}_{1}}{\Gamma(q+1)}\left[q\left(\sum_{i=1}^{m-2} \delta_{i} \sigma_{i}^{q-1}+\left|\rho_{4}\right|\right)+\sum_{j=1}^{p-2} \gamma_{j}\left(\eta_{j}^{q}-\xi_{j}^{q}\right)\right] \\
& +\frac{1}{\mu \Gamma(q)}\left(\widehat{\Omega}_{2}+1\right)\left(1-e^{-\mu}\right) \\
& \widehat{\lambda}=\lambda-\frac{1}{\mu \Gamma(q)}\left(1-e^{-\mu}\right), \\
& \widehat{\phi}_{i}=\max _{t \in[0,1]}\left|\phi_{i}(t)\right|=\frac{1}{\mu|\Delta|}\left[\mu\left|B_{2} \alpha_{i}+\mu B_{1} \delta_{i}\right|+\left(1-e^{-\mu}\right)\left|A_{2} \alpha_{i}+\mu A_{1} \delta_{i}\right|\right] \text {, } \\
& \widehat{\psi}_{j}=\max _{t \in[0,1]}\left|\psi_{j}(t)\right|=\frac{1}{\mu|\Delta|}\left[\mu\left|B_{2} r_{j}+\mu B_{1} \gamma_{j}\right|+\left|\left(A_{2} r_{j}+\mu A_{1} \gamma_{j}\right)\right|\left(1-e^{-\mu}\right)\right], \\
& \widehat{\Omega}_{1}=\max _{t \in[0,1]}\left|\Omega_{1}(t)\right|=\frac{1}{\mu|\Delta|}\left[\mu\left|B_{1}\right|+\left|A_{1}\right|\left(1-e^{-\mu}\right)\right], \\
& \widehat{\Omega}_{2}=\max _{t \in[0,1]}\left|\Omega_{2}(t)\right|=\frac{1}{\mu|\Delta|}\left[\mu\left|\rho_{4} \mu B_{1}+\rho_{2} B_{2}\right|+\left(1-e^{-\mu}\right)\left|\rho_{4} \mu A_{1}+\rho_{2} A_{2}\right|\right] ; \\
& \lambda_{1}=\frac{1}{\mu \Gamma(q)} \sum_{i=1}^{m-2} \widehat{\phi}_{i}^{\prime} \sigma_{i}^{q-1}\left(1-e^{-\mu \sigma_{i}}\right)+\frac{1}{\mu \Gamma(q+1)} \sum_{j=1}^{p-2} \widehat{\psi}_{j}^{\prime}\left(\eta_{j}^{q}-\xi_{j}^{q}\right)\left(1-e^{-\mu \eta_{j}}\right) \\
& +\frac{\widehat{\Omega}_{1}^{\prime}}{\Gamma(q+1)}\left[q\left(\sum_{i=1}^{m-2} \delta_{i} \sigma_{i}^{q-1}+\left|\rho_{4}\right|\right)+\sum_{j=1}^{p-2} \gamma_{j}\left(\eta_{j}^{q}-\xi_{j}^{q}\right)\right] \\
& +\frac{1}{\mu \Gamma(q)} \widehat{\Omega}_{2}^{\prime}\left(1-e^{-\mu}\right)+\frac{2-e^{-\mu}}{\Gamma(q)}, \\
& \widehat{\lambda}_{1}=\lambda_{1}-\frac{2-e^{-\mu}}{\Gamma(q)} \text {, } \\
& \widehat{\phi}_{i}^{\prime}=\max _{t \in[0,1]}\left|\phi_{i}^{\prime}(t)\right|=\frac{1}{|\Delta|}\left[\mu\left|B_{2} \alpha_{i}+\mu B_{1} \delta_{i}\right|+\left|A_{2} \alpha_{i}+\mu A_{1} \delta_{i}\right|\right], \\
& \widehat{\psi}_{j}^{\prime}=\max _{t \in[0,1]}\left|\psi_{i}^{\prime}(t)\right|=\frac{1}{|\Delta|}\left[\mu\left|B_{2} r_{j}+\mu B_{1} \gamma_{j}\right|+\left|A_{2} r_{j}+\mu A_{1} \gamma_{j}\right|\right] \text {, } \\
& \widehat{\Omega}_{1}^{\prime}=\max _{t \in[0,1]}\left|\Omega_{1}^{\prime}(t)\right|=\frac{1}{|\Delta|}\left[\mu\left|B_{1}\right|+\left|A_{1}\right|\right], \\
& \widehat{\Omega}_{2}^{\prime}=\max _{t \in[0,1]}\left|\Omega_{2}^{\prime}(t)\right|=\frac{1}{|\Delta|}\left[\mu\left|\rho_{4} \mu B_{1}+\rho_{2} B_{2}\right|+\left|\rho_{4} \mu A_{1}+\rho_{2} A_{2}\right|\right] .
\end{aligned}
$$


In the following, for brevity, we use the notation

$$
\widehat{f}(x(\theta))=f\left(\theta, x(\theta),{ }^{c} D^{\kappa} x(\theta)\right)
$$

\subsection{Existence result via Krasnoselskii's fixed point theorem}

Here we present an existence result for the problem (1.1) and (1.3), which relies on Krasnoselskii's fixed point theorem [31].

Theorem 3.1 Let $f:[0,1] \times \mathbb{R} \times \mathbb{R} \rightarrow \mathbb{R}$ be a continuous function satisfying the conditions:

$\left(A_{1}\right)$ There exists a constant $L>0$ such that $\left|f\left(t, x_{1}, y_{1}\right)-f\left(t, x_{2}, y_{2}\right)\right| \leq L\left(\left|x_{1}-x_{2}\right|+\left|y_{1}-y_{2}\right|\right)$ for all $t \in[0,1], x_{i}, y_{i} \in \mathbb{R}, i=1,2$;

$\left(B_{1}\right)$ There exists a function $m \in C\left([0,1], \mathbb{R}^{+}\right)$such that $|f(t, x, y)| \leq m(t)$ for all $(t, x, y) \in$ $[0,1] \times \mathbb{R} \times \mathbb{R}$.

Then the problem (1.1) and (1.3) has at least one solution on $[0,1]$ provided that

$$
L\left(\widehat{\lambda}+\frac{1}{\Gamma(2-\kappa)} \widehat{\lambda}_{1}\right)<1
$$

where $\widehat{\lambda}, \widehat{\lambda}_{1}$ are given by (3.4) and (3.6), respectively.

Proof For $\rho>\|m\|\left(\lambda+\frac{\lambda_{1}}{\Gamma(2-\kappa)}\right)$, we consider the closed ball $B_{\rho}=\left\{x \in X:\|x\|_{X} \leq \rho\right\}$ and introduce the operators $A$ and $B$ on $B_{\rho}$ as follows:

$$
\begin{aligned}
(A x)(t)= & \sum_{i=1}^{m-2} \phi_{i}(t) \int_{0}^{\sigma_{i}} e^{\mu\left(s-\sigma_{i}\right)}\left(I^{q-1} \widehat{f}(x(s))\right) d s \\
& +\sum_{j=1}^{p-2} \psi_{j}(t) \int_{\xi_{j}}^{\eta_{j}}\left(\int_{0}^{s} e^{\mu(m-s)}\left(I^{q-1} \widehat{f}(x(m))\right) d m\right) d s \\
& +\Omega_{1}(t)\left[\sum _ { i = 1 } ^ { m - 2 } \delta _ { i } \left(I^{q-1} \widehat{f}\left(x\left(\sigma_{i}\right)\right)\right.\right. \\
& \left.+\sum_{j=1}^{p-2} \gamma_{j} \int_{\xi_{j}}^{\eta_{j}}\left(I^{q-1} \widehat{f}(x(s))\right) d s-\rho_{4} I^{q-1} \widehat{f}(x(1))\right] \\
& +\Omega_{2}(t) \int_{0}^{1} e^{\mu(s-1)}\left(I^{q-1} \widehat{f}(x(s))\right) d s, \quad t \in[0,1], \\
(B x)(t)= & \int_{0}^{t} e^{\mu(s-t)}\left(I^{q-1} \widehat{f}(x(s))\right) d s, \quad t \in[0,1] .
\end{aligned}
$$

For any $x, y \in B_{\rho}$, it is straightforward to show that

$$
\|(\mathfrak{H} x)\|_{X}=\|A x+B x\|_{X} \leq\|m\|\left(\lambda+\frac{\lambda_{1}}{\Gamma(2-\kappa)}\right)<\rho,
$$

which implies that $A x+B y \in B_{\rho}$. Also, the operator $B$ is completely continuous. Indeed, $B$ is uniformly bounded on $B_{r}$ as

$$
\|B x\|_{X} \leq \frac{1-e^{-\mu}}{\mu \Gamma(q)}+\frac{2-e^{-\mu}}{\Gamma(2-\kappa) \Gamma(q)} .
$$


Observe that

$$
\begin{aligned}
& \left|B x\left(t_{2}\right)-B x\left(t_{1}\right)\right| \\
& \quad \leq\left|\int_{0}^{t_{1}}\left[e^{\mu\left(s-t_{2}\right)}-e^{\mu\left(s-t_{1}\right)}\right]\left(I^{q-1}|\widehat{f}(x(s))|\right) d s+\int_{t_{1}}^{t_{2}} e^{\mu\left(s-t_{2}\right)}\left(I^{q-1}|\widehat{f}(x(s))|\right) d s\right| \\
& \quad \leq\|m\|\left|\int_{0}^{t_{1}}\left[e^{\mu\left(s-t_{2}\right)}-e^{\mu\left(s-t_{1}\right)}\right]\left(I^{q-1} 1\right) d s+\int_{t_{1}}^{t_{2}} e^{\mu\left(s-t_{2}\right)}\left(I^{q-1} 1\right) d s\right| \rightarrow 0, \quad \text { as } t_{1} \rightarrow t_{2} .
\end{aligned}
$$

Also

$$
\begin{aligned}
& \left|B x^{\prime}\left(t_{2}\right)-B x^{\prime}\left(t_{1}\right)\right| \\
& \leq\|m\| \mu\left|\int_{0}^{t_{1}}\left[e^{\mu\left(s-t_{2}\right)}-e^{\mu\left(s-t_{1}\right)}\right]\left(I^{q-1} 1\right) d s+\int_{t_{1}}^{t_{2}} e^{\mu\left(s-t_{2}\right)}\left(I^{q-1} 1\right) d s\right| \\
& \quad+\frac{\|m\|}{\Gamma(q-1)}\left|\int_{0}^{t_{1}}\left[\left(t_{2}-s\right)^{q-2}-\left(t_{1}-s\right)^{q-2}\right] d s+\int_{t_{1}}^{t_{2}}\left(t_{2}-s\right)^{q-2} d s\right| \rightarrow 0,
\end{aligned}
$$

and

$$
\begin{aligned}
\left|{ }^{c} D^{\kappa} B x\left(t_{2}\right)-{ }^{c} D^{\kappa} B x\left(t_{1}\right)\right| & \leq \int_{0}^{t} \frac{(t-s)^{-\kappa}}{\Gamma(1-\kappa)}\left|B x^{\prime}\left(t_{2}\right)-B x^{\prime}\left(t_{1}\right)\right| d s \\
& \leq \frac{1}{\Gamma(2-\kappa)}\left|B x^{\prime}\left(t_{2}\right)-B x^{\prime}\left(t_{1}\right)\right| \rightarrow 0,
\end{aligned}
$$

as $t_{1} \rightarrow t_{2}$ independent of $x$. So $B$ is equicontinuous. Using assumption $\left(A_{1}\right)$ and condition (3.8), it is easy to establish that operator $A$ is a contraction. Thus the hypotheses of Krasnoselskii's fixed point theorem [31] hold true, and consequently we deduce by its conclusion that the problem (1.1) and (1.3) has at least one solution on [0,1]. This completes the proof.

\subsection{Uniqueness of solutions}

Here we establish the uniqueness of solutions for the problem (1.1) and (1.3) by applying a fixed point theorem due to Banach.

Theorem 3.2 Let assumption $\left(A_{1}\right)$ be satisfied and

$$
L\left(\lambda+\frac{\lambda_{1}}{\Gamma(2-q)}\right)<1,
$$

where $\lambda$ and $\lambda_{1}$ are given by (3.3) and (3.5), respectively. Then there exists a unique solution for the problem (1.1) and (1.3) on $[0,1]$.

Proof Let us define $\sup _{t \in[0,1]}|f(t, 0,0)|=M$ and select

$$
\bar{r} \geq \frac{M\left(\lambda+\frac{\lambda_{1}}{\Gamma(2-q)}\right)}{1-L\left(\lambda+\frac{\lambda_{1}}{\Gamma(2-q)}\right)}
$$

to show that $\mathfrak{H} B_{\bar{r}} \subset B_{\bar{r}}$, where $B_{\bar{r}}=\left\{x \in X:\|x\|_{X} \leq \bar{r}\right\}$ and $\mathfrak{H}$ is defined by (3.1). 
Using condition $\left(A_{1}\right)$, we have

$$
\begin{aligned}
|\widehat{f}(x(\theta))| & =\left|f\left(\theta, x(\theta),{ }^{c} D^{\kappa} x(\theta)\right)\right|=\left|f\left(\theta, x(\theta),{ }^{c} D^{\kappa} x(\theta)\right)-f(\theta, 0,0)\right|+|f(\theta, 0,0)| \\
& \leq L\left(\|x\|+\left\|{ }^{c} D^{\kappa} x\right\|\right)+M \leq L\|x\|_{X}+M \leq L \bar{r}+M .
\end{aligned}
$$

Then, for $x \in B_{\bar{r}}$, we obtain

$$
\begin{aligned}
\|(\mathfrak{H} x)\|= & \sup _{t \in[0,1]}|(\mathfrak{H} x)(t)| \\
\leq & \sup _{t \in[0,1]}\left\{\left|\sum_{i=1}^{m-2}\right| \phi_{i}(t) \mid \int_{0}^{\sigma_{i}} e^{\mu\left(s-\sigma_{i}\right)}\left(I^{q-1}|\widehat{f}(x(s))|\right) d s\right. \\
& +\sum_{j=1}^{p-2}\left|\psi_{j}(t)\right| \int_{\xi_{j}}^{\eta_{j}}\left(\int_{0}^{s} e^{\mu(m-s)}\left(I^{q-1}|\widehat{f}(x(m))|\right) d m\right) d s \\
& +\left|\Omega_{1}(t)\right|\left[\sum _ { i = 1 } ^ { m - 2 } | \delta _ { i } | \left(I^{q-1} \mid \widehat{f}\left(x\left(\sigma_{i}\right) \mid\right)\right.\right. \\
& \left.+\sum_{j=1}^{p-2}\left|\gamma_{j}\right| \int_{\xi_{j}}^{\eta_{j}}\left(I^{q-1}|\widehat{f}(x(s))|\right) d s+\left|\rho_{4}\right| I^{q-1}|\widehat{f}(x(1))|\right] \\
& \left.+\left|\Omega_{2}(t)\right| \int_{0}^{1} e^{\mu(s-1)}\left(I^{q-1}|\widehat{f}(x(s))|\right) d s+\int_{0}^{t} e^{\mu(s-t)}\left(I^{q-1}|\widehat{f}(x(s))|\right) d s\right\} \\
\leq & (L \bar{r}+M)\left\{\sum_{i=1}^{m-2} \widehat{\phi}_{i} \int_{0}^{\sigma_{i}} e^{\mu\left(s-\sigma_{i}\right)}\left(I^{q-1} 1\right) d s\right. \\
& +\sum_{j=1}^{p-2} \widehat{\psi_{j}} \int_{\xi_{j}}^{\eta_{j}}\left(\int_{0}^{s} e^{\mu(m-s)}\left(I^{q-1} 1\right) d m\right) d s \\
& +\widehat{\Omega}_{1}\left[\sum_{i=1}^{m-2} \delta_{i}\left(I^{q-1} 1\right)+\sum_{j=1}^{p} e^{\mu-2} \gamma_{j} \int_{\xi_{j}}^{\eta_{j}}\left(I^{q-1} 1\right) d s-\rho_{4} I^{q-1} 1\right] \\
& +M .
\end{aligned}
$$

Also we have

$$
\begin{aligned}
\left|(\mathfrak{H} x)^{\prime}(t)\right| \leq & \sum_{i=1}^{m-2}\left|\phi_{i}^{\prime}(t)\right| \int_{0}^{\sigma_{i}} e^{\mu\left(s-\sigma_{i}\right)}\left(I^{q-1} \widehat{f}(x(s)) \mid\right) d s \\
& +\sum_{j=1}^{p-2}\left|\psi_{j}^{\prime}(t)\right| \int_{\xi_{j}}^{\eta_{j}}\left(\int_{0}^{s} e^{\mu(m-s)}\left(I^{q-1}|\widehat{f}(x(m))|\right) d m\right) d s \\
& +\left|\Omega_{1}^{\prime}(t)\right|\left[\sum_{i=1}^{m-2}\left|\delta_{i}\right|\left(I^{q-1}\left|\widehat{f}\left(x\left(\sigma_{i}\right)\right)\right|\right)\right.
\end{aligned}
$$




$$
\begin{aligned}
& \left.+\sum_{j=1}^{p-2}\left|\gamma_{j}\right| \int_{\xi_{j}}^{\eta_{j}}\left(I^{q-1}|\widehat{f}(x(s))|\right) d s+\left|\rho_{4}\right| I^{q-1}|\widehat{f}(x(1))|\right] \\
& +\left|\Omega_{2}^{\prime}(t)\right| \int_{0}^{1} e^{\mu(s-1)}\left(I^{q-1}|\widehat{f}(x(s))|\right) d s \\
& +\mu \int_{0}^{t} e^{\mu(s-t)}\left(I^{q-1} \widehat{f}(x(s)) \mid\right) d s+I^{q-1}|\widehat{f}(x(s))| \\
& \leq(L \bar{r}+M) \lambda_{1} .
\end{aligned}
$$

Using the above inequality in the definition of Caputo fractional derivative with $0<\kappa \leq 1$, we get

$$
\begin{aligned}
\left|{ }^{c} D^{\kappa}(\mathfrak{H} x)(t)\right| & \leq \int_{0}^{t} \frac{(t-s)^{-\kappa}}{\Gamma(1-\kappa)}\left|(\mathfrak{H} x)^{\prime}(s)\right| \leq(L \bar{r}+M) \lambda_{1} \int_{0}^{t} \frac{(t-s)^{-\kappa}}{\Gamma(1-\kappa)} d s \\
& \leq \frac{1}{\Gamma(2-\kappa)}(L \bar{r}+M) \lambda_{1} .
\end{aligned}
$$

Hence

$$
\|(\mathfrak{H} x)\|_{X}=\|(\mathfrak{H} x)\|+\left\|{ }^{c} D^{\kappa}(\mathfrak{H} x)\right\| \leq(L \bar{r}+M) \lambda+\frac{1}{\Gamma(2-\kappa)}(L \bar{r}+M) \lambda_{1}<\bar{r},
$$

which clearly shows that $\mathfrak{H} x \in B_{\bar{r}}$ for any $x \in B_{\bar{r}}$. Thus $\mathfrak{H} B_{\bar{r}} \subset B_{\bar{r}}$.

Now, for $x, y \in X$ and for each $t \in[0,1]$, we have

$$
\begin{aligned}
& |(\mathfrak{H} x)(t)-(\mathfrak{H} y)(t)| \\
& \leq \sum_{i=1}^{m-2}\left|\phi_{i}(t)\right| \int_{0}^{\sigma_{i}} e^{\mu\left(s-\sigma_{i}\right)}\left(I^{q-1}|\widehat{f}(x(s))-\widehat{f}(y(s))|\right) d s \\
& \quad+\sum_{j=1}^{p-2}\left|\psi_{j}(t)\right| \int_{\xi_{j}}^{\eta_{j}}\left(\int_{0}^{s} e^{\mu(m-s)}\left(I^{q-1}|\widehat{f}(x(m))-\widehat{f}(y(m))|\right) d m\right) d s \\
& \quad+\left|\Omega_{1}(t)\right|\left[\sum_{i=1}^{m-2}\left|\delta_{i}\right|\left(I^{q-1}\left|\widehat{f}\left(x\left(\sigma_{i}\right)\right)-\widehat{f}\left(y\left(\sigma_{i}\right)\right)\right|\right)\right. \\
& \quad+\sum_{j=1}^{p-2}\left|\gamma_{j}\right| \int_{\xi_{j}}^{\eta_{j}}\left(I^{q-1}|\widehat{f}(x(s))-\widehat{f}(y(s))|\right) d s \\
& \left.\quad+\left|\rho_{4}\right| I^{q-1}|\widehat{f}(x(1))-\widehat{f}(y(1))|\right]+\left|\Omega_{2}(t)\right| \int_{0}^{1} e^{\mu(s-1)}\left(I^{q-1}|\widehat{f}(x(s))-\widehat{f}(y(s))|\right) d s \\
& \quad+\int_{0}^{t} e^{\mu(s-t)}\left(I^{q-1}|\widehat{f}(x(s))-\widehat{f}(y(s))|\right) d s \\
& \leq L \lambda\|x-y\|_{X} .
\end{aligned}
$$

Also

$$
\begin{aligned}
& \left|(\mathfrak{H} x)^{\prime}(t)-(\mathfrak{H} y)^{\prime}(t)\right| \\
& \quad \leq \sum_{i=1}^{m-2}\left|\phi_{i}^{\prime}(t)\right| \int_{0}^{\sigma_{i}} e^{\mu\left(s-\sigma_{i}\right)}\left(I^{q-1}|\widehat{f}(x(s))-\widehat{f}(y(s))|\right) d s
\end{aligned}
$$




$$
\begin{aligned}
& +\sum_{j=1}^{p-2}\left|\psi_{j}^{\prime}(t)\right| \int_{\xi_{j}}^{\eta_{j}}\left(\int_{0}^{s} e^{\mu(m-s)}\left(I^{q-1} \widehat{f}(x(m))-\widehat{f}(y(m)) \mid\right) d m\right) d s \\
& +\left|\Omega_{1}^{\prime}(t)\right|\left[\sum_{i=1}^{m-2}\left|\delta_{i}\right|\left(I^{q-1}\left|\widehat{f}\left(x\left(\sigma_{i}\right)\right)-\widehat{f}\left(y\left(\sigma_{i}\right)\right)\right|\right)\right. \\
& \left.+\sum_{j=1}^{p-2}\left|\gamma_{j}\right| \int_{\xi_{j}}^{\eta_{j}}\left(I^{q-1}|\widehat{f}(x(s))-\widehat{f}(y(s))|\right) d s+\left|\rho_{4}\right| I^{q-1}|\widehat{f}(x(1))-\widehat{f}(y(1))|\right] \\
& +\left|\Omega_{2}^{\prime}(t)\right| \int_{0}^{1} e^{\mu(s-1)}\left(I^{q-1} \widehat{f}(x(s))-\widehat{f}(y(s)) \mid\right) d s \\
& +\mu \int_{0}^{t} e^{\mu(s-t)}\left(I^{q-1}|\widehat{f}(x(s))-\widehat{f}(y(s))|\right) d s+I^{q-1} \widehat{f}(x(s))-\widehat{f}(y(s)) \mid \\
& \leq L \lambda_{1}\|x-y\|_{X},
\end{aligned}
$$

and moreover,

$$
\left|{ }^{c} D^{\kappa}(\mathfrak{H} x)(t)-{ }^{c} D^{\kappa}(\mathfrak{H} y)(t)\right| \leq \frac{1}{\Gamma(2-\kappa)} L \lambda_{1}\|x-y\|_{X} .
$$

Consequently, we have

$$
\|(\mathfrak{H} x)-(\mathfrak{H} y)\|_{X} \leq L\left(\lambda+\frac{\lambda_{1}}{\Gamma(2-q)}\right)\|x-y\|_{X},
$$

which shows that $\mathfrak{H}$ is a contraction by condition (3.11). Thus the operator $\mathfrak{H}$ has a unique fixed point by Banach fixed point theorem, which corresponds to a unique solution of the problem (1.1) and (1.3) on $[0,1]$. This completes the proof.

\subsection{An example}

Consider the following nonlinear sequential fractional differential equation:

$$
\begin{aligned}
\left(D^{7 / 5}+D^{2 / 5}\right) x(t)= & \frac{1}{t^{2}+25} \\
& +\frac{e^{-t}}{t^{4}+16}\left(\tan ^{-1}(x(t))+\frac{\left|{ }^{c} D^{1 / 2} x(t)\right|}{\left(1+\left|{ }^{c} D^{1 / 2} x(t)\right|\right)}\right), \quad t \in[0,1],
\end{aligned}
$$

supplemented with the integro-multipoint boundary conditions:

$$
\begin{aligned}
& \rho_{1} x(0)+\rho_{2} x(1)=\sum_{i=1}^{3} \alpha_{i} x\left(\sigma_{i}\right)+\sum_{j=1}^{5} r_{j} \int_{\xi_{j}}^{\eta_{i}} x(s) d s, \\
& \rho_{3} x^{\prime}(0)+\rho_{4} x^{\prime}(1)=\sum_{i=1}^{3} \delta_{i} x^{\prime}\left(\sigma_{i}\right)+\sum_{j=1}^{5} \gamma_{j} \int_{\xi_{j}}^{\eta_{j}} x^{\prime}(s) d s .
\end{aligned}
$$

Here $q=7 / 5, \kappa=1 / 2, \mu=1, m=5, p=7, \sigma_{1}=1 / 18, \sigma_{2}=1 / 9, \sigma_{3}=1 / 6, \xi_{1}=1 / 5, \eta_{1}=1 / 4$, $\xi_{2}=3 / 10, \eta_{2}=7 / 20, \xi_{3}=2 / 5, \eta_{3}=9 / 20, \xi_{4}=1 / 2, \eta_{4}=11 / 20, \xi_{5}=3 / 5, \eta_{5}=13 / 20, \alpha_{1}=1$, $\alpha_{2}=1 / 2, \alpha_{3}=1, \delta_{1}=3 / 4, \delta_{2}=1, \delta_{3}=3 / 2, r_{1}=1 / 3, r_{2}=2 / 3, r_{3}=1, r_{4}=-1, r_{5}=2, \gamma_{1}=-1 / 2$, $\gamma_{2}=1 / 2, \gamma_{3}=2, \gamma_{4}=1, \gamma_{5}=3 / 2, \rho_{1}=1 / 2, \rho_{2}=3 / 4, \rho_{3}=-1, \rho_{4}=2$. Using the given data, 
we find that $\Delta=4.3972, \lambda=2.4121, \widehat{\lambda}=1.6997, \lambda_{1}=4.1293, \widehat{\lambda}_{1}=2.2898\left(\Delta, \lambda, \widehat{\lambda}, \lambda_{1}\right.$ and $\widehat{\lambda}_{1}$ are respectively given by (2.7), (3.3), (3.4), (3.5), and (3.6)). Further we have

$$
m(t)=\frac{1}{t^{2}+25}+\frac{(\pi+2) e^{-t}}{2\left(t^{4}+16\right)}, \quad L=\frac{1}{16}, \quad L\left(\widehat{\lambda}+\frac{1}{\Gamma(2-\kappa)} \widehat{\lambda}_{1}\right) \approx 0.2677<1 .
$$

Clearly, the hypothesis of Theorem 3.1 holds true. In consequence, there exists a solution to the problem (3.12)-(3.13) on $[0,1]$ by Theorem 3.1. Also

$$
L\left(\lambda+\frac{\lambda_{1}}{\Gamma(2-\kappa)}\right) \approx 0.4420<1
$$

which implies that the problem (3.12)-(3.13) has a unique solution on $[0,1]$ by Theorem 3.2.

\section{The case of inclusions}

In this section, we investigate the existence of solutions for the multivalued (inclusion) boundary value problem (1.2) and (1.3). Our first result deals with the case when the multivalued map $F$ has convex values, while the second result is concerned with the nonconvex multivalued maps.

For each $x \in C([0,1], \mathbb{R})$, the set

$$
S_{F, x}=\left\{f \in L^{1}([0,1], \mathbb{R}): f(t) \in F\left(t, x(t),{ }^{c} D^{\kappa} x(t)\right) \text { for a.e. } t \in[0,1]\right\}
$$

is known as the set of selections of the multivalued map $F$.

\subsection{The case of upper semicontinuous (convex-valued) maps}

Here we present an existence result for the problem (1.2) and (1.3) when the multivalued map $F$ is convex-valued. We make use of nonlinear alternative for Kakutani maps [32] to derive the desired result.

Theorem 4.1 Assume that:

$\left(C_{1}\right) F:[0,1] \times \mathbb{R}^{2} \rightarrow \mathcal{P}(\mathbb{R})$ is $L^{1}$-Carathéodory and has nonempty, compact, and convex values;

$\left(C_{2}\right)$ There exist a function $g \in C\left([0,1], \mathbb{R}^{+}\right)$with $\|g\|=\sup _{t \in[0,1]}|g(t)|$ and nondecreasing function $Q: \mathbb{R}^{+} \rightarrow \mathbb{R}^{+}$such that

$$
\begin{aligned}
\|F(t, x, y)\|_{\mathcal{P}} & :=\sup \{|w|: w \in F(t, x, y)\} \\
& \leq g(t) Q(\|x\|+\|y\|), \quad \forall(t, x, y) \in[0,1] \times \mathbb{R} \times \mathbb{R} ;
\end{aligned}
$$

$\left(C_{3}\right)$ There exists a constant $K>0$ such that

$$
\frac{K}{\|g\| Q(K)\left(\lambda+\frac{\lambda_{1}}{\Gamma(2-\kappa)}\right)}>1
$$

where $\lambda, \lambda_{1}$ are are given by (3.3) and (3.5), respectively.

Then the problem (1.2)-(1.3) has at least one solution on $[0,1]$. 
Proof We transform the problem (1.2)-(1.3) into a fixed point problem. Consider the multivalued map: $N: X \rightarrow \mathcal{P}(X)$ defined by

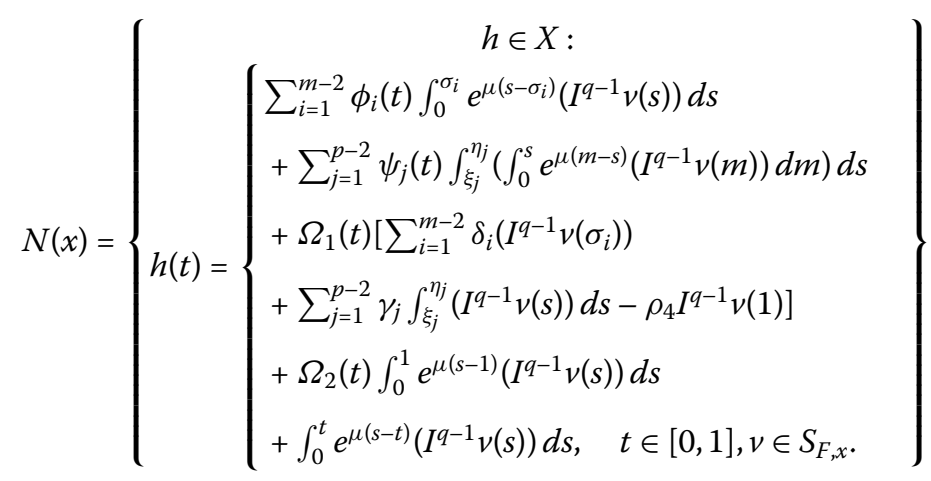

It is clear that the fixed points of $N$ are solutions of problem (1.2)-(1.3). Now we proceed to show that operator $N$ satisfies all the conditions of the nonlinear alternative for Kakutani maps [32]. The proof is given in several steps.

Step 1. $N(x)$ is convex for each $x \in X$.

Indeed, if $h_{1}, h_{2}$ belong to $N(x)$, then there exist $v_{1}, v_{2} \in S_{F, x}$ such that for each $t \in[0,1]$, we have

$$
\begin{aligned}
h_{k}(t)= & \sum_{i=1}^{m-2} \phi_{i}(t) \int_{0}^{\sigma_{i}} e^{\mu\left(s-\sigma_{i}\right)}\left(I^{q-1} v_{k}(s)\right) d s \\
& +\sum_{j=1}^{p-2} \psi_{j}(t) \int_{\xi_{j}}^{\eta_{j}}\left(\int_{0}^{s} e^{\mu(m-s)}\left(I^{q-1} v_{k}(m)\right) d m\right) d s+\Omega_{1}(t)\left[\sum_{i=1}^{m-2} \delta_{i}\left(I^{q-1} v_{k}\left(\sigma_{i}\right)\right)\right. \\
& \left.+\sum_{j=1}^{p-2} \gamma_{j} \int_{\xi_{j}}^{\eta_{j}}\left(I^{q-1} v_{k}(s)\right) d s-\rho_{4} I^{q-1} v_{k}(1)\right] \\
& +\Omega_{2}(t) \int_{0}^{1} e^{\mu(s-1)}\left(I^{q-1} v_{k}(s)\right) d s+\int_{0}^{t} e^{\mu(s-t)}\left(I^{q-1} v_{k}(s)\right) d s, \quad k=1,2 .
\end{aligned}
$$

Let $0 \leq \mu \leq 1$. Then for each $t \in[0,1]$, we have

$$
\begin{aligned}
& {\left[\mu h_{1}+(1-\mu) h_{2}\right](t)} \\
& =\sum_{i=1}^{m-2} \phi_{i}(t) \int_{0}^{\sigma_{i}} e^{\mu\left(s-\sigma_{i}\right)}\left(I^{q-1}\left[\mu v_{1}(s)+(1-\mu) v_{2}(s)\right]\right) d s \\
& \quad+\sum_{j=1}^{p-2} \psi_{j}(t) \int_{\xi_{j}}^{\eta_{j}}\left(\int_{0}^{s} e^{\mu(m-s)}\left(I^{q-1}\left[\mu v_{1}(m)+(1-\mu) v_{2}(m)\right]\right) d m\right) d s \\
& \quad+\Omega_{1}(t)\left[\sum_{i=1}^{m-2} \delta_{i}\left(I^{q-1}\left[\mu v_{1}\left(\sigma_{i}\right)+(1-\mu) v_{2}\left(\sigma_{i}\right)\right]\right)\right. \\
& \quad+\sum_{j=1}^{p-2} \gamma_{j} \int_{\xi_{j}}^{\eta_{j}}\left(I^{q-1}\left[\mu v_{1}(s)+(1-\mu) v_{2}(s)\right]\right) d s
\end{aligned}
$$




$$
\begin{aligned}
& \left.-\rho_{4} I^{q-1}\left[\mu v_{1}(1)+(1-\mu) v_{2}(1)\right]\right] \\
& +\Omega_{2}(t) \int_{0}^{1} e^{\mu(s-1)}\left(I^{q-1}\left[\mu v_{1}(s)+(1-\mu) v_{2}(s)\right]\right) d s \\
& +\int_{0}^{t} e^{\mu(s-t)}\left(I^{q-1}\left[\mu v_{1}(s)+(1-\mu) v_{2}(s)\right]\right) d s .
\end{aligned}
$$

Since $F$ has convex values $\left(S_{F, x}\right.$ is convex),

$$
\mu h_{1}+(1-\mu) h_{2} \in N(x) .
$$

Step 2. $N(x)$ maps bounded sets (balls) into bounded sets in $X$.

For a positive number $r$, let $\mathcal{B}_{r}=\left\{x \in X:\|x\|_{X} \leq r\right\}$ be a bounded set in $X$. Then, for $h \in$ $N(x), x \in B_{r}$, there exists $v \in S_{F, x}$ such that

$$
\begin{aligned}
h(t)= & \sum_{i=1}^{m-2} \phi_{i}(t) \int_{0}^{\sigma_{i}} e^{\mu\left(s-\sigma_{i}\right)}\left(I^{q-1} v(s)\right) d s \\
& +\sum_{j=1}^{p-2} \psi_{j}(t) \int_{\xi_{j}}^{\eta_{j}}\left(\int_{0}^{s} e^{\mu(m-s)}\left(I^{q-1} v(m)\right) d m\right) d s+\Omega_{1}(t)\left[\sum_{i=1}^{m-2} \delta_{i}\left(I^{q-1} v\left(\sigma_{i}\right)\right)\right. \\
& \left.+\sum_{j=1}^{p-2} \gamma_{j} \int_{\xi_{j}}^{\eta_{j}}\left(I^{q-1} v(s)\right) d s-\rho_{4} I^{q-1} v(1)\right] \\
& +\Omega_{2}(t) \int_{0}^{1} e^{\mu(s-1)}\left(I^{q-1} v(s)\right) d s+\int_{0}^{t} e^{\mu(s-t)}\left(I^{q-1} v(s)\right) d s
\end{aligned}
$$

for some $v \in S_{F, x_{r}}$. Then, for $t \in[0,1]$, we have

$$
\begin{aligned}
& |h(t)| \leq \sum_{i=1}^{m-2} \widehat{\phi}_{i} \int_{0}^{\sigma_{i}} e^{\mu\left(s-\sigma_{i}\right)}\left(I^{q-1}|v(s)|\right) d s \\
& +\sum_{j=1}^{p-2} \widehat{\psi}_{j} \int_{\xi_{j}}^{\eta_{j}}\left(\int_{0}^{s} e^{\mu(m-s)}\left(I^{q-1}|v(m)|\right) d m\right) d s \\
& +\widehat{\Omega}_{1}\left[\sum_{i=1}^{m-2} \delta_{i}\left(I^{q-1}|v(s)|\right)+\sum_{j=1}^{p-2} \gamma_{j} \int_{\xi_{j}}^{\eta_{j}}\left(I^{q-1}|v(1)|\right) d s-\rho_{4} I^{q-1}|v(1)|\right] \\
& +\widehat{\Omega}_{2} \int_{0}^{1} e^{\mu(s-1)}\left(I^{q-1}|v(s)|\right) d s+\int_{0}^{t} e^{\mu(s-t)}\left(I^{q-1}|v(s)|\right) d s \\
& \leq\|g\| Q\left(\|x\|+\left\|{ }^{c} D^{\kappa} x\right\|\right)\left\{\sum_{i=1}^{m-2} \widehat{\phi}_{i} \int_{0}^{\sigma_{i}} e^{\mu\left(s-\sigma_{i}\right)}\left(I^{q-1} 1\right) d s\right. \\
& +\sum_{j=1}^{p-2} \widehat{\psi}_{j} \int_{\xi_{j}}^{\eta_{j}}\left(\int_{0}^{s} e^{\mu(m-s)}\left(I^{q-1} 1\right) d m\right) d s \\
& +\widehat{\Omega}_{1}\left[\sum_{i=1}^{m-2} \delta_{i}\left(I^{q-1} 1\right)+\sum_{j=1}^{p-2} \gamma_{j} \int_{\xi_{j}}^{\eta_{j}}\left(I^{q-1} 1\right) d s-\rho_{4} I^{q-1} 1\right]
\end{aligned}
$$




$$
\begin{aligned}
& \left.\quad+\widehat{\Omega}_{2} \int_{0}^{1} e^{\mu(s-1)}\left(I^{q-1} 1\right) d s+\int_{0}^{t} e^{\mu(s-t)}\left(I^{q-1} 1\right) d s\right\} \\
& \leq\|g\| Q\left(\|x\|_{X}\right) \lambda,
\end{aligned}
$$

which, when taking the norm for $t \in[0,1]$, yields $\|h\| \leq\|g\| Q(r) \lambda$. Also we have

$$
\begin{aligned}
\left|h^{\prime}(t)\right| \leq & \sum_{i=1}^{m-2}\left|\phi_{i}^{\prime}(t)\right| \int_{0}^{\sigma_{i}} e^{\mu\left(s-\sigma_{i}\right)}\left(I^{q-1}|v(s)|\right) d s \\
& +\sum_{j=1}^{p-2}\left|\psi_{j}^{\prime}(t)\right| \int_{\xi_{j}}^{\eta_{j}}\left(\int_{0}^{s} e^{\mu(m-s)}\left(I^{q-1}|v(m)|\right) d m\right) d s \\
& +\left|\Omega_{1}^{\prime}(t)\right|\left[\sum_{i=1}^{m-2}\left|\delta_{i}\right|\left(I^{q-1}\left|v\left(\sigma_{i}\right)\right|\right)\right. \\
& \left.+\sum_{j=1}^{p-2}\left|\gamma_{j}\right| \int_{\xi_{j}}^{\eta_{j}}\left(I^{q-1}|v(s)|\right) d s+\left|\rho_{4}\right| I^{q-1}|v(1)|\right] \\
& +\left|\Omega_{2}^{\prime}(t)\right| \int_{0}^{1} e^{\mu(s-1)}\left(I^{q-1}|v(s)|\right) d s \\
& +\mu \int_{0}^{t} e^{\mu(s-t)}\left(I^{q-1}|v(s)|\right) d s+I^{q-1}|v(s)| \\
\leq & \|g\| Q\left(\|x\|_{X}\right) \lambda_{1} \leq\|g\| Q(r) \lambda_{1} .
\end{aligned}
$$

By definition of Caputo fractional derivative with $0<\kappa \leq 1$, we get

$$
\left|{ }^{c} D^{\kappa} h(t)\right| \leq \int_{0}^{t} \frac{(t-s)^{-\kappa}}{\Gamma(1-\kappa)}\left|h^{\prime}(t)\right| \leq\|g\| Q(r) \lambda_{1} \int_{0}^{t} \frac{(t-s)^{-\kappa}}{\Gamma(1-\kappa)} d s \leq \frac{1}{\Gamma(2-\kappa)}\|g\| Q(r) \lambda_{1} .
$$

Hence

$$
\|h\|_{X}=\|h\|+\left\|{ }^{c} D^{\kappa} h\right\| \leq\|g\| Q(r)\left(\lambda+\frac{\lambda_{1}}{\Gamma(2-\kappa)}\right) .
$$

Step 3. $N$ maps bounded sets into equicontinuous sets of $X$.

Next we show that $N$ maps bounded sets into equicontinuous sets of $X$. Let $t_{1}, t_{2} \in[0,1]$ with $t_{1}<t_{2}$ and $y \in \mathcal{B}_{r}$, where $\mathcal{B}_{r}$ is a bounded set of $X$. Then we obtain

$$
\begin{aligned}
\left|h\left(t_{2}\right)-h\left(t_{1}\right)\right| \leq & \sum_{i=1}^{m-2}\left|\phi_{i}\left(t_{2}\right)-\phi_{i}\left(t_{1}\right)\right| \int_{0}^{\sigma_{i}} e^{\mu\left(s-\sigma_{i}\right)}\left(I^{q-1}|v(s)|\right) d s \\
& +\sum_{j=1}^{p-2}\left|\psi_{j}\left(t_{2}\right)-\psi_{j}\left(t_{1}\right)\right| \int_{\xi_{j}}^{\eta_{j}}\left(\int_{0}^{s} e^{\mu(m-s)}\left(I^{q-1}|v(m)|\right) d m\right) d s \\
& +\left|\Omega_{1}\left(t_{2}\right)-\Omega_{1}\left(t_{1}\right)\right|\left[\sum_{i=1}^{m-2}\left|\delta_{i}\right|\left(I^{q-1}\left|v\left(\sigma_{i}\right)\right|\right)+\sum_{j=1}^{p-2}\left|\gamma_{j}\right| \int_{\xi_{j}}^{\eta_{j}}\left(I^{q-1}|v(s)|\right) d s\right. \\
& \left.+\left|\rho_{4}\right| I^{q-1}|v(1)|\right]+\left|\Omega_{2}\left(t_{2}\right)-\Omega_{2}\left(t_{1}\right)\right| \int_{0}^{1} e^{\mu(s-1)}\left(I^{q-1}|v(s)|\right) d s
\end{aligned}
$$




$$
\begin{aligned}
& +\left|\int_{0}^{t_{1}}\left[e^{\mu\left(s-t_{2}\right)}-e^{\mu\left(s-t_{1}\right)}\right]\left(I^{q-1}|v(s)|\right) d s+\int_{t_{1}}^{t_{2}} e^{\mu\left(s-t_{2}\right)}\left(I^{q-1}|v(s)|\right) d s\right| \\
& \leq\|g\| Q(r)\left\{\sum_{i=1}^{m-2}\left|\phi_{i}\left(t_{2}\right)-\phi_{i}\left(t_{1}\right)\right| \int_{0}^{\sigma_{i}} e^{\mu\left(s-\sigma_{i}\right)}\left(I^{q-1} 1\right) d s\right. \\
& +\sum_{j=1}^{p-2}\left|\psi_{j}\left(t_{2}\right)-\psi_{j}\left(t_{1}\right)\right| \int_{\xi_{j}}^{\eta_{j}}\left(\int_{0}^{s} e^{\mu(m-s)}\left(I^{q-1} 1\right) d m\right) d s \\
& +\left|\Omega_{1}\left(t_{2}\right)-\Omega_{1}\left(t_{1}\right)\right|\left[\sum_{i=1}^{m-2}\left|\delta_{i}\right|\left(I^{q-1} 1\right)+\sum_{j=1}^{p-2}\left|\gamma_{j}\right| \int_{\xi_{j}}^{\eta_{j}}\left(I^{q-1} 1\right) d s\right. \\
& \left.+\left|\rho_{4}\right| I^{q-1} 1\right]+\left|\Omega_{2}\left(t_{2}\right)-\Omega_{2}\left(t_{1}\right)\right| \int_{0}^{1} e^{\mu(s-1)}\left(I^{q-1}\right) d s \\
& \left.+\left|\int_{0}^{t_{1}}\left[e^{\mu\left(s-t_{2}\right)}-e^{\mu\left(s-t_{1}\right)}\right]\left(I^{q-1} 1\right) d s+\int_{t_{1}}^{t_{2}} e^{\mu\left(s-t_{2}\right)}\left(I^{q-1} 1\right) d s\right|\right\}, \\
& \left|h^{\prime}\left(t_{2}\right)-h^{\prime}\left(t_{1}\right)\right| \leq\|g\| Q(r)\left\{\sum_{i=1}^{m-2}\left|\phi_{i}^{\prime}\left(t_{2}\right)-\phi_{i}^{\prime}\left(t_{1}\right)\right| \int_{0}^{\sigma_{i}} e^{\mu\left(s-\sigma_{i}\right)}\left(I^{q-1} 1\right) d s\right. \\
& +\sum_{j=1}^{p-2}\left|\psi_{j}^{\prime}\left(t_{2}\right)-\psi_{j}^{\prime}\left(t_{1}\right)\right| \int_{\xi_{j}}^{\eta_{j}}\left(\int_{0}^{s} e^{\mu(m-s)}\left(I^{q-1} 1\right) d m\right) d s \\
& +\left|\Omega_{1}^{\prime}\left(t_{2}\right)-\Omega_{1}^{\prime}\left(t_{1}\right)\right|\left[\sum_{i=1}^{m-2}\left|\delta_{i}\right|\left(I^{q-1} 1\right)+\sum_{j=1}^{p-2}\left|\gamma_{j}\right| \int_{\xi_{j}}^{\eta_{j}}\left(I^{q-1} 1\right) d s\right. \\
& \left.+\left|\rho_{4}\right| I^{q-1} 1\right]+\left|\Omega_{2}^{\prime}\left(t_{2}\right)-\Omega_{2}^{\prime}\left(t_{1}\right)\right| \int_{0}^{1} e^{\mu(s-1)}\left(I^{q-1} 1\right) d s \\
& +\mu\left|\int_{0}^{t_{1}}\left[e^{\mu\left(s-t_{2}\right)}-e^{\mu\left(s-t_{1}\right)}\right]\left(I^{q-1} 1\right) d s+\int_{t_{1}}^{t_{2}} e^{\mu\left(s-t_{2}\right)}\left(I^{q-1} 1\right) d s\right| \\
& \left.+\frac{1}{\Gamma(q-1)}\left|\int_{0}^{t_{1}}\left[\left(t_{2}-s\right)^{q-2}-\left(t_{1}-s\right)^{q-2}\right] d s+\int_{t_{1}}^{t_{2}}\left(t_{2}-s\right)^{q-2} d s\right|\right\},
\end{aligned}
$$

and

$$
\begin{aligned}
\left|{ }^{c} D^{\kappa} h\left(t_{2}\right)-{ }^{c} D^{\kappa} h\left(t_{1}\right)\right| & \leq \int_{0}^{t} \frac{(t-s)^{-\kappa}}{\Gamma(1-\kappa)}\left|h^{\prime}\left(t_{2}\right)-h^{\prime}\left(t_{1}\right)\right| d s \\
& \leq \frac{1}{\Gamma(2-\kappa)}\left|h^{\prime}\left(t_{2}\right)-h^{\prime}\left(t_{1}\right)\right| .
\end{aligned}
$$

Obviously, the right-hand sides of the above inequalities tend to zero independently of $x \in \mathcal{B}_{r}$ as $t_{2}-t_{1} \rightarrow 0$. As $N$ satisfies the above assumptions, it follows by the Arzelá-Ascoli theorem that $N: X \rightarrow X$ is completely continuous.

By virtue of Proposition 1.2 in [33], it is enough to establish that operator $N: X \rightarrow X$ has a closed graph, which will imply that $N$ is u.s.c. as it is already shown to be completely continuous. This is done in our next step.

Step 4. $N$ has a closed graph. 
Let $x_{n} \rightarrow x_{*}, h_{n} \in N\left(x_{n}\right)$, and $h_{n} \rightarrow h_{*}$. We need to show that $h_{*} \in N\left(x_{*}\right)$. Now $h_{n} \in N\left(x_{n}\right)$ implies that there exists $v_{n} \in S_{F, x_{n}}$ such that for each $t \in[0,1]$,

$$
\begin{aligned}
h_{n}(t)= & \sum_{i=1}^{m-2} \phi_{i}(t) \int_{0}^{\sigma_{i}} e^{\mu\left(s-\sigma_{i}\right)}\left(I^{q-1} v_{n}(s)\right) d s \\
& +\sum_{j=1}^{p-2} \psi_{j}(t) \int_{\xi_{j}}^{\eta_{j}}\left(\int_{0}^{s} e^{\mu(m-s)}\left(I^{q-1} v_{n}(m)\right) d m\right) d s \\
& +\Omega_{1}(t)\left[\sum_{i=1}^{m-2} \delta_{i}\left(I^{q-1} v_{n}\left(\sigma_{i}\right)\right)+\sum_{j=1}^{p-2} \gamma_{j} \int_{\xi_{j}}^{\eta_{j}}\left(I^{q-1} v_{n}(s)\right) d s-\rho_{4} I^{q-1} v_{n}(1)\right] \\
& +\Omega_{2}(t) \int_{0}^{1} e^{\mu(s-1)}\left(I^{q-1} v_{n}(s)\right) d s+\int_{0}^{t} e^{\mu(s-t)}\left(I^{q-1} v_{n}(s)\right) d s .
\end{aligned}
$$

We must show that there exists $v_{*} \in S_{F, x_{*}}$ such that for each $t \in[0,1]$,

$$
\begin{aligned}
h_{*}(t)= & \sum_{i=1}^{m-2} \phi_{i}(t) \int_{0}^{\sigma_{i}} e^{\mu\left(s-\sigma_{i}\right)}\left(I^{q-1} v_{*}(s)\right) d s \\
& +\sum_{j=1}^{p-2} \psi_{j}(t) \int_{\xi_{j}}^{\eta_{j}}\left(\int_{0}^{s} e^{\mu(m-s)}\left(I^{q-1} v_{*}(m)\right) d m\right) d s+\Omega_{1}(t)\left[\sum_{i=1}^{m-2} \delta_{i}\left(I^{q-1} v_{*}\left(\sigma_{i}\right)\right)\right. \\
& \left.+\sum_{j=1}^{p-2} \gamma_{j} \int_{\xi_{j}}^{\eta_{j}}\left(I^{q-1} v_{*}(s)\right) d s-\rho_{4} I^{q-1} v_{*}(1)\right] \\
& +\Omega_{2}(t) \int_{0}^{1} e^{\mu(s-1)}\left(I^{q-1} v_{*}(s)\right) d s+\int_{0}^{t} e^{\mu(s-t)}\left(I^{q-1} v_{*}(s)\right) d s .
\end{aligned}
$$

Consider the continuous linear operator $\Theta: L^{1}([0,1], \mathbb{R}) \rightarrow C([0,1], \mathbb{R})$ given by

$$
\begin{aligned}
v \mapsto \Theta(v)(t)= & \sum_{i=1}^{m-2} \phi_{i}(t) \int_{0}^{\sigma_{i}} e^{\mu\left(s-\sigma_{i}\right)}\left(I^{q-1} v(s)\right) d s \\
& +\sum_{j=1}^{p-2} \psi_{j}(t) \int_{\xi_{j}}^{\eta_{j}}\left(\int_{0}^{s} e^{\mu(m-s)}\left(I^{q-1} v(m)\right) d m\right) d s \\
& +\Omega_{1}(t)\left[\sum_{i=1}^{m-2} \delta_{i}\left(I^{q-1} v\left(\sigma_{i}\right)\right)+\sum_{j=1}^{p-2} \gamma_{j} \int_{\xi_{j}}^{\eta_{j}}\left(I^{q-1} v(s)\right) d s-\rho_{4} I^{q-1} v(1)\right] \\
& +\Omega_{2}(t) \int_{0}^{1} e^{\mu(s-1)}\left(I^{q-1} v(s)\right) d s+\int_{0}^{t} e^{\mu(s-t)}\left(I^{q-1} v(s)\right) d s .
\end{aligned}
$$

Since $\left\|h_{n}-h_{*}\right\|_{X} \rightarrow 0$ as $n \rightarrow \infty$, it follows from a closed graph result obtained in [34] that $\Theta \circ S_{F, x}$ is a closed graph operator. Moreover, we have $h_{n} \in \Theta\left(S_{F, x_{n}}\right)$. Since $x_{n} \rightarrow x_{*}$, we obtain

$$
h_{*}(t)=\sum_{i=1}^{m-2} \phi_{i}(t) \int_{0}^{\sigma_{i}} e^{\mu\left(s-\sigma_{i}\right)}\left(I^{q-1} v_{*}(s)\right) d s
$$




$$
\begin{aligned}
& +\sum_{j=1}^{p-2} \psi_{j}(t) \int_{\xi_{j}}^{\eta_{j}}\left(\int_{0}^{s} e^{\mu(m-s)}\left(I^{q-1} v_{*}(m)\right) d m\right) d s \\
& +\Omega_{1}(t)\left[\sum_{i=1}^{m-2} \delta_{i}\left(I^{q-1} \nu_{*}\left(\sigma_{i}\right)\right)+\sum_{j=1}^{p-2} \gamma_{j} \int_{\xi_{j}}^{\eta_{j}}\left(I^{q-1} v_{*}(s)\right) d s-\rho_{4} I^{q-1} v_{*}(1)\right] \\
& +\Omega_{2}(t) \int_{0}^{1} e^{\mu(s-1)}\left(I^{q-1} v_{*}(s)\right) d s+\int_{0}^{t} e^{\mu(s-t)}\left(I^{q-1} v_{*}(s)\right) d s
\end{aligned}
$$

for some $v_{*} \in S_{F, x_{*}}$

Step 5. We show there exists an open set $U \subseteq C(J, \mathbb{R})$ with $x \notin \theta N(x)$ for any $\theta \in(0,1)$

and all $x \in \partial U$.

Let $\theta \in(0,1)$ and $x \in \theta N(x)$. Then there exists $v \in L^{1}([0,1], \mathbb{R})$ with $v \in S_{F, x}$ such that, for $t \in J$, we have

$$
\begin{aligned}
x(t)= & \theta \sum_{i=1}^{m-2} \phi_{i}(t) \int_{0}^{\sigma_{i}} e^{\mu\left(s-\sigma_{i}\right)}\left(I^{q-1} v(s)\right) d s \\
& +\theta \sum_{j=1}^{p-2} \psi_{j}(t) \int_{\xi_{j}}^{\eta_{j}}\left(\int_{0}^{s} e^{\mu(m-s)}\left(I^{q-1} v(m)\right) d m\right) d s+\theta \Omega_{1}(t)\left[\sum_{i=1}^{m-2} \delta_{i}\left(I^{q-1} v\left(\sigma_{i}\right)\right)\right. \\
& \left.+\sum_{j=1}^{p-2} \gamma_{j} \int_{\xi_{j}}^{\eta_{j}}\left(I^{q-1} v(s)\right) d s-\rho_{4} I^{q-1} v(1)\right] \\
& +\theta \Omega_{2}(t) \int_{0}^{1} e^{\mu(s-1)}\left(I^{q-1} v(s)\right) d s+\int_{0}^{t} e^{\mu(s-t)}\left(I^{q-1} v(s)\right) d s .
\end{aligned}
$$

As in the first step, we can find that

$$
\frac{\|x\|_{X}}{\|g\| Q\left(\|x\|_{X}\right)\left(\lambda+\frac{\lambda_{1}}{\Gamma(2-\kappa)}\right)} \leq 1
$$

By condition $\left(C_{3}\right)$, there does not exist any solution $x$ such that $\|x\|_{X} \neq K$. Let us introduce a set $W=\left\{x \in X:\|x\|_{X}<K\right\}$. The operator $N: \bar{W} \rightarrow X$ is continuous and completely continuous. From the choice of $W$, there is no $w \in \partial W$ such that $w=\theta N(w)$ for some $\theta \in(0,1)$. In consequence, we deduce by the nonlinear alternative for Kakutani maps [32] that operator $N$ has a fixed point $w \in \bar{W}$ which is a solution of the problem (1.2) and (1.3). The proof is completed.

\subsection{The case of Lipschitz maps}

Now we prove the existence of solutions for the boundary value problem (1.2) and (1.3) with a nonconvex valued right-hand side by applying a fixed point theorem for multivalued maps due to Covitz and Nadler [35].

Let $(X, d)$ be a metric space induced from the normed space $(X ;\|\cdot\|)$. Consider $H_{d}$ : $\mathcal{P}(X) \times \mathcal{P}(X) \rightarrow \mathbb{R} \cup\{\infty\}$ given by

$$
H_{d}(U, V)=\max \left\{\sup _{u \in U} d(u, V), \sup _{v \in V} d(U, v)\right\}
$$

where $d(U, v)=\inf _{u \in U} d(u ; v)$ and $d(u, V)=\inf _{v \in V} d(u ; v)$. Then $\left(\mathcal{P}_{c l, b}(X), H_{d}\right)$ is a metric space (see [36]), where $\mathcal{P}_{c l, b}(X)=\{\mathcal{Y} \in \mathcal{P}(X): \mathcal{Y}$ is closed and bounded $\}$. 
Definition 4.2 A multivalued operator $N: X \rightarrow \mathcal{P}_{c l}(X)$ is called (a) $\theta$-Lipschitz if and only if there exists $\theta>0$ such that $H_{d}(N(x), N(y)) \leq \theta d(x, y)$ for each $x, y \in X$; and (b) a contraction if and only if it is $\theta$-Lipschitz with $\theta<1$.

Lemma 4.3 ([35]) Let $(X, d)$ be a complete metric space and $\mathcal{P}_{c l}(X)=\{\mathcal{Y} \in \mathcal{P}(X)$ : $\mathcal{Y}$ is closed\}. If $N: X \rightarrow \mathcal{P}_{c l}(X)$ is a contraction, then Fix $N \neq \emptyset$.

Theorem 4.4 Assume that the following conditions hold:

$\left(K_{1}\right) \quad F:[0,1] \times \mathbb{R}^{2} \rightarrow \mathcal{P}_{c p}(\mathbb{R})$ is such that $F(\cdot, x, y):[0,1] \rightarrow \mathcal{P}_{c p}(\mathbb{R})$ is measurable for each $x, y \in \mathbb{R}$

$\left(K_{2}\right) H_{d}(F(t, x, y), F(t, \bar{x}, \bar{y})) \leq m(t)(|x-\bar{x}|+|y-\bar{y}|)$ for almost all $t \in[0,1]$ and $x, \bar{x}, y, \bar{y} \in \mathbb{R}$ with $m \in C\left([0,1], \mathbb{R}^{+}\right)$and $d(0, F(t, 0,0)) \leq m(t)$ for almost all $t \in[0,1]$.

Then the inclusion problem (1.2) and (1.3) has at least one solution on $[0,1]$ if

$$
\|m\|\left(\lambda+\frac{\lambda_{1}}{\Gamma(2-\kappa)}\right)<1
$$

where $\lambda, \lambda_{1}$ are given by (3.3) and (3.5), respectively.

Proof In order to show that the operator $N: C([0,1], \mathbb{R}) \rightarrow \mathcal{P}(C([0,1], \mathbb{R}))$ (introduced in the beginning of the proof of Theorem 4.1) satisfies the assumptions of Lemma 4.3, we proceed as follows.

Step I. $N(x)$ is nonempty and closed for every $v \in S_{F, x}$.

Notice that $S_{F, x} \neq \emptyset$ for each $x$ by assumption $\left(K_{2}\right)$, and thus we can find a measurable selection for $F$ (see [37, Theorem III.6]). In order to show that $N(x) \in \mathcal{P}_{c l}(X)$ for each $x \in X$, let $\left\{u_{n}\right\}_{n \geq 0} \in N(x)$ be such that $u_{n} \rightarrow u(n \rightarrow \infty)$ in $X$. Then $u \in C(J, \mathbb{R})$ and there exists $v_{n} \in S_{F, x_{n}}$ such that, for each $t \in[0,1]$, we have

$$
\begin{aligned}
u_{n}(t)= & \sum_{i=1}^{m-2} \phi_{i}(t) \int_{0}^{\sigma_{i}} e^{\mu\left(s-\sigma_{i}\right)}\left(I^{q-1} v_{n}(s)\right) d s \\
& +\sum_{j=1}^{p-2} \psi_{j}(t) \int_{\xi_{j}}^{\eta_{j}}\left(\int_{0}^{s} e^{\mu(m-s)}\left(I^{q-1} v_{n}(m)\right) d m\right) d s+\Omega_{1}(t)\left[\sum_{i=1}^{m-2} \delta_{i}\left(I^{q-1} v_{n}\left(\sigma_{i}\right)\right)\right. \\
& \left.+\sum_{j=1}^{p-2} \gamma_{j} \int_{\xi_{j}}^{\eta_{j}}\left(I^{q-1} v_{n}(s)\right) d s-\rho_{4} I^{q-1} v_{n}(1)\right] \\
& +\Omega_{2}(t) \int_{0}^{1} e^{\mu(s-1)}\left(I^{q-1} v_{n}(s)\right) d s+\int_{0}^{t} e^{\mu(s-t)}\left(I^{q-1} v_{n}(s)\right) d s .
\end{aligned}
$$

Since $F$ has compact values, one can pass onto a subsequence (if necessary) to find that $v_{n}$ converges to $v$ in $L^{1}([0,1], \mathbb{R})$. Thus $v \in S_{F, x}$ and for each $t \in[0,1]$, we have

$$
\begin{aligned}
u_{n}(t) \rightarrow u(t)= & \sum_{i=1}^{m-2} \phi_{i}(t) \int_{0}^{\sigma_{i}} e^{\mu\left(s-\sigma_{i}\right)}\left(I^{q-1} v(s)\right) d s \\
& +\sum_{j=1}^{p-2} \psi_{j}(t) \int_{\xi_{j}}^{\eta_{j}}\left(\int_{0}^{s} e^{\mu(m-s)}\left(I^{q-1} v(m)\right) d m\right) d s
\end{aligned}
$$




$$
\begin{aligned}
& +\Omega_{1}(t)\left[\sum_{i=1}^{m-2} \delta_{i}\left(I^{q-1} v\left(\sigma_{i}\right)\right)+\sum_{j=1}^{p-2} \gamma_{j} \int_{\xi_{j}}^{\eta_{j}}\left(I^{q-1} v(s)\right) d s-\rho_{4} I^{q-1} v(1)\right] \\
& +\Omega_{2}(t) \int_{0}^{1} e^{\mu(s-1)}\left(I^{q-1} v(s)\right) d s+\int_{0}^{t} e^{\mu(s-t)}\left(I^{q-1} v(s)\right) d s,
\end{aligned}
$$

which implies that $u \in N(x)$.

Step II. There exists $\bar{\theta}<1$ ( $\bar{\theta}$ is given by (4.3)) such that

$$
H_{d}(N(x), N(\bar{x})) \leq \bar{\theta}\|x-\bar{x}\|_{X} \quad \text { for each } x, \bar{x} \in C([0,1], \mathbb{R}) .
$$

Let $x, \bar{x} \in X$ and $h_{1} \in N(x)$. Then we can find $v_{1}(t) \in F(t, x(t), y(t))$ such that, for each $t \in$ $[0,1]$,

$$
\begin{aligned}
h_{1}(t)= & \sum_{i=1}^{m-2} \phi_{i}(t) \int_{0}^{\sigma_{i}} e^{\mu\left(s-\sigma_{i}\right)}\left(I^{q-1} v_{1}(s)\right) d s \\
& +\sum_{j=1}^{p-2} \psi_{j}(t) \int_{\xi_{j}}^{\eta_{j}}\left(\int_{0}^{s} e^{\mu(m-s)}\left(I^{q-1} v_{1}(m)\right) d m\right) d s+\Omega_{1}(t)\left[\sum_{i=1}^{m-2} \delta_{i}\left(I^{q-1} v_{1}\left(\sigma_{i}\right)\right)\right. \\
& \left.+\sum_{j=1}^{p-2} \gamma_{j} \int_{\xi_{j}}^{\eta_{j}}\left(I^{q-1} v_{1}(s)\right) d s-\rho_{4} I^{q-1} v_{1}(1)\right] \\
& +\Omega_{2}(t) \int_{0}^{1} e^{\mu(s-1)}\left(I^{q-1} v_{1}(s)\right) d s+\int_{0}^{t} e^{\mu(s-t)}\left(I^{q-1} v_{1}(s)\right) d s .
\end{aligned}
$$

By $\left(K_{2}\right)$, we have

$$
H_{d}(F(t, x, y), F(t, \bar{x}, \bar{y}) \leq m(t)(|x(t)-\bar{x}(t)|+|y(t)-\bar{y}(t)|) .
$$

So there exists $w(t) \in F(t, \bar{x}(t), \bar{y}(t))$ such that

$$
\left|v_{1}(t)-w\right| \leq m(t)(|x(t)-\bar{x}(t)|+|y(t)-\bar{y}(t)|), \quad t \in[0,1] .
$$

Define $\mathcal{V}:[0,1] \rightarrow \mathcal{P}(\mathbb{R})$ by

$$
\mathcal{V}(t)=\left\{w \in \mathbb{R}:\left|v_{1}(t)-w\right| \leq m(t)(|x(t)-\bar{x}(t)|+|y(t)-\bar{y}(t)|)\right\} .
$$

In view of the fact that the multivalued operator $\mathcal{V}(t) \cap F(t, \bar{x}(t), \bar{y}(t))$ is measurable (see [37, Proposition III.4]), we can find a function $v_{2}(t)$ which is a measurable selection for $\mathcal{V}$ and such that $v_{2}(t) \in F(t, \bar{x}(t), \bar{y}(t))$. Then, for each $t \in[0,1]$, we have $\left|v_{1}(t)-v_{2}(t)\right| \leq$ $m(t)(|x(t)-\bar{x}(t)|+|y(t)-\bar{y}(t)|)$. For each $t \in[0,1]$, we define

$$
\begin{aligned}
h_{2}(t)= & \sum_{i=1}^{m-2} \phi_{i}(t) \int_{0}^{\sigma_{i}} e^{\mu\left(s-\sigma_{i}\right)}\left(I^{q-1} v_{2}(s)\right) d s \\
& +\sum_{j=1}^{p-2} \psi_{j}(t) \int_{\xi_{j}}^{\eta_{j}}\left(\int_{0}^{s} e^{\mu(m-s)}\left(I^{q-1} v_{2}(m)\right) d m\right) d s+\Omega_{1}(t)\left[\sum_{i=1}^{m-2} \delta_{i}\left(I^{q-1} v_{2}\left(\sigma_{i}\right)\right)\right.
\end{aligned}
$$




$$
\begin{aligned}
& \left.+\sum_{j=1}^{p-2} \gamma_{j} \int_{\xi_{j}}^{\eta_{j}}\left(I^{q-1} v_{2}(s)\right) d s-\rho_{4} I^{q-1} v_{2}(1)\right] \\
& +\Omega_{2}(t) \int_{0}^{1} e^{\mu(s-1)}\left(I^{q-1} v_{2}(s)\right) d s+\int_{0}^{t} e^{\mu(s-t)}\left(I^{q-1} v_{2}(s)\right) d s .
\end{aligned}
$$

Thus,

$$
\begin{aligned}
& \left|h_{1}(t)-h_{2}(t)\right| \leq \sum_{i=1}^{m-2}\left|\phi_{i}(t)\right| \int_{0}^{\sigma_{i}} e^{\mu\left(s-\sigma_{i}\right)}\left(I^{q-1}\left|v_{2}(s)-v_{1}(s)\right|\right) d s \\
& +\sum_{j=1}^{p-2}\left|\psi_{j}(t)\right| \int_{\xi_{j}}^{\eta_{j}}\left(\int_{0}^{s} e^{\mu(m-s)}\left(I^{q-1}\left|\nu_{2}(m)-v_{1}(m)\right|\right) d m\right) d s \\
& +\left|\Omega_{1}(t)\right|\left[\sum_{i=1}^{m-2}\left|\delta_{i}\right|\left(I^{q-1}\left|v_{2}(\sigma)-v_{1}(\sigma)\right|\right)\right. \\
& \left.+\sum_{j=1}^{p-2}\left|\gamma_{j}\right| \int_{\xi_{j}}^{\eta_{j}}\left(I^{q-1}\left|v_{2}(s)-v_{1}(s)\right|\right) d s-\rho_{4} I^{q-1}\left|v_{2}(1)-v_{1}(1)\right|\right] \\
& +\left|\Omega_{2}(t)\right| \int_{0}^{1} e^{\mu(s-1)}\left(I^{q-1}\left|v_{2}(s)-v_{1}(s)\right|\right) d s \\
& +\int_{0}^{t} e^{\mu(s-t)}\left(I^{q-1}\left|v_{2}(s)-v_{1}(s)\right|\right) d s \\
& \leq\left\{\sum_{i=1}^{m-2} \widehat{\phi}_{i} \int_{0}^{\sigma_{i}} e^{\mu\left(s-\sigma_{i}\right)}\left(I^{q-1} 1\right) d s\right. \\
& +\sum_{j=1}^{p-2} \widehat{\psi}_{j} \int_{\xi_{j}}^{\eta_{j}}\left(\int_{0}^{s} e^{\mu(m-s)}\left(I^{q-1} 1\right) d m\right) d s \\
& +\widehat{\Omega}_{1}\left[\sum_{i=1}^{m-2} \delta_{i}\left(I^{q-1} 1\right)+\sum_{j=1}^{p-2} \gamma_{j} \int_{\xi_{j}}^{\eta_{j}}\left(I^{q-1} 1\right) d s-\rho_{4} I^{q-1} 1\right] \\
& \left.+\widehat{\Omega}_{2} \int_{0}^{1} e^{\mu(s-1)}\left(I^{q-1} 1\right) d s+\int_{0}^{t} e^{\mu(s-t)}\left(I^{q-1} 1\right) d s\right\}\|m\|\|x-\bar{x}\| \\
& \leq \lambda\|m\|\|x-\bar{x}\|_{X} .
\end{aligned}
$$

Hence $\left\|h_{1}-h_{2}\right\| \leq \lambda\|m\|\|x-\bar{x}\|_{X}$. In a similar manner, we obtain

$$
\begin{aligned}
\left|h_{1}^{\prime}(t)-h_{2}^{\prime}(t)\right| \leq & \left\{\sum_{i=1}^{m-2}\left|\phi_{i}^{\prime}(t)\right| \int_{0}^{\sigma_{i}} e^{\mu\left(s-\sigma_{i}\right)}\left(I^{q-1} 1\right) d s\right. \\
& +\sum_{j=1}^{p-2}\left|\psi_{j}^{\prime}(t)\right| \int_{\xi_{j}}^{\eta_{j}}\left(\int_{0}^{s} e^{\mu(m-s)}\left(I^{q-1} 1\right) d m\right) d s+\left|\Omega_{1}^{\prime}(t)\right|\left[\sum_{i=1}^{m-2}\left|\delta_{i}\right|\left(I^{q-1} 1\right)\right. \\
& \left.+\sum_{j=1}^{p-2}\left|\gamma_{j}\right| \int_{\xi_{j}}^{\eta_{j}}\left(I^{q-1} 1\right) d s+\left|\rho_{4}\right| I^{q-1} 1\right]+\left|\Omega_{2}^{\prime}(t)\right| \int_{0}^{1} e^{\mu(s-1)}\left(I^{q-1} 1\right) d s
\end{aligned}
$$




$$
\begin{aligned}
& \left.\quad+\mu \int_{0}^{t} e^{\mu(s-t)}\left(I^{q-1} 1\right) d s+I^{q-1} 1\right\}\|m\|\|x-\bar{x}\|_{X} \\
& \leq \lambda_{1}\|m\|\|x-\bar{x}\|_{X},
\end{aligned}
$$

and

$$
\left|{ }^{c} D^{\kappa} h_{1}(t)-{ }^{c} D^{\kappa} h_{2}(t)\right| \leq \int_{0}^{t} \frac{(t-s)^{-\kappa}}{\Gamma(1-\kappa)}\left|h_{1}^{\prime}(s)-h_{2}^{\prime}(s)\right| d s \leq \frac{1}{\Gamma(2-\kappa)} \lambda_{1}\|m\|\|x-\bar{x}\|_{X} .
$$

Thus

$$
\left\|h_{1}-h_{2}\right\|_{X} \leq\|m\|\left(\lambda+\frac{\lambda_{1}}{\Gamma(2-\kappa)}\right)\|x-\bar{x}\|_{X}
$$

Analogously, interchanging the roles of $x$ and $\bar{x}$, we obtain

$$
H_{d}(N(x), N(\bar{x})) \leq\|m\|\left(\lambda+\frac{\lambda_{1}}{\Gamma(2-\kappa)}\right)\|x-\bar{x}\|_{X} .
$$

From the foregoing arguments, we deduce that $N$ is a contraction. Thus it follows by Lemma 4.3 that $N$ has a fixed point $x$ which is a solution of (1.2) and (1.3). This completes the proof.

\subsection{Examples}

Consider the sequential fractional differential inclusion

$$
\left({ }^{c} D^{7 / 5}+D^{2 / 5}\right) x(t) \in F\left(t, x(t),{ }^{c} D^{1 / 2} x(t)\right), \quad t \in[0,1],
$$

equipped with conditions (3.13).

In order to illustrate Theorem 4.1, we take

$$
\begin{aligned}
F\left(t, x(t),{ }^{c} D^{1 / 2} x(t)\right)= & {\left[\frac{1}{\sqrt{t^{2}+144}}\left(\frac{1}{3} \sin (x(t))+\frac{1}{2} \frac{\left|{ }^{c} D^{1 / 2} x(t)\right|}{\left(1+\left|{ }^{c} D^{1 / 2} x(t)\right|\right)}+\frac{1}{2}\right),\right.} \\
& \left.\frac{1}{(t+15)}\left(\frac{1}{16} e^{-x^{4}(t)}+\frac{1}{5} \sin \left({ }^{c} D^{1 / 2} x(t)\right)+\frac{1}{2}\right)\right] .
\end{aligned}
$$

It is easy to find that $g(t)=\frac{1}{\sqrt{t^{2}+144}}$ with $\|g\|=1 / 12, \Omega(K)=(K+3) / 3$. By condition $\left(C_{3}\right)$, we find that $K>0.7333$. Thus all the assumptions of Theorem 4.1 hold, and consequently the inclusion (4.4) equipped with boundary conditions (3.13) and $F$ given by (4.5) has a solution on $[0,1]$.

For illustrating Theorem 4.4, we consider the following multivalued map:

$$
\begin{aligned}
F\left(t, x(t),{ }^{c} D^{1 / 2} x(t)\right)= & {\left[\frac{1}{t+10} x(t)+\frac{1}{t^{2}+15} \cos \left(D^{1 / 2} x(t)\right),\right.} \\
& \left.\frac{1}{t^{2}+25} \tan ^{-1}(x(t))+\frac{1}{t^{2}+49}{ }^{c} D^{1 / 2} x(t)+\frac{1}{98}\right] .
\end{aligned}
$$

By condition $\left(D_{2}\right)$, we get $m(t)=1 /(t+10)$ with $\|m\|=1 / 10$. Moreover,

$$
\|m\|\left(\lambda+\frac{\lambda_{1}}{\Gamma(2-\kappa)}\right) \approx 0.70715<1
$$


(the values of $\lambda$ and $\lambda_{1}$ are taken from Sect. 3.1). Clearly, all the hypotheses of Theorem 4.4 are satisfied. Therefore there exists at least one solution on $[0,1]$ for the inclusion (4.4) with $F$ given by (4.6), equipped with boundary conditions (3.13).

\section{Conclusions}

In this paper, we have addressed new problems of sequential fractional differential equations and inclusions supplemented with nonlocal integro-multipoint boundary conditions. The single- and multivalued maps involved in the given problems depend on the unknown function, together with its lower-order fractional derivative. Our results are not only new in the given configuration but also yield several new results as special cases by fixing the parameters appearing in the problems. Some of these results are mentioned below.

- Our results correspond to purely nonlocal multipoint and multistrip boundary conditions if we take $\rho_{i}=0(i=1,2,3,4)$ in the given problems.

- For $\rho_{1}=\rho_{3}=0, \rho_{2}=\rho_{4}=1$, we obtain the results for terminal nonlocal multipoint and multistrip conditions:

$$
x(1)=\sum_{i=1}^{m-2} \alpha_{i} x\left(\sigma_{i}\right)+\sum_{j=1}^{p-2} r_{j} \int_{\xi_{j}}^{\eta_{i}} x(s) d s, \quad x^{\prime}(1)=\sum_{i=1}^{m-2} \delta_{i} x^{\prime}\left(\sigma_{i}\right)+\sum_{j=1}^{p-2} \gamma_{j} \int_{\xi_{j}}^{\eta_{j}} x^{\prime}(s) d s .
$$

- The results for sequential fractional differential equations and inclusions equipped with periodic/antiperiodic type boundary conditions of the form: $x(0)=-\left(\rho_{2} / \rho_{1}\right) x(1)$, $x^{\prime}(0)=-\left(\rho_{4} / \rho_{3}\right) x^{\prime}(1)$ follow by fixing $r_{j}=\gamma_{j}=\alpha_{j}=\delta_{j}=0, j=1, \ldots, p$. Further, the results for antiperiodic boundary conditions can be recorded by taking $\rho_{2} / \rho_{1}=1=\rho_{4} / \rho_{3}$.

- The results associated with nonseparated nonlocal multipoint and multistrip conditions can be obtained by letting $r_{j}=0=\gamma_{j}, j=1, \ldots, p$ and $\alpha_{j}=0=\delta_{j}, j=1, \ldots, p$, respectively.

In the context of sequential fractional differential equations and inclusions together with nonlocal integro-multipoint boundary conditions, the present work is quite versatile in nature and significantly contributes to the existing literature on the topic. Moreover, several new results follow as special cases of the present ones.

\section{Acknowledgements}

This project was funded by the Deanship of Scientific Research (DSR), King Abdulaziz University, Jeddah, Saudi Arabia under grant no. (RG-25-130-38). The authors, therefore, acknowledge with thanks DSR technical and financial support. The authors also thank the reviewers for their constructive remarks on our paper.

\section{Funding}

This project was funded by the Deanship of Scientific Research (DSR), King Abdulaziz University, Jeddah, Saudi Arabia under grant no. (RG-25-130-38).

\section{Abbreviations}

Not applicable.

Availability of data and materials

Not applicable.

\section{Competing interests}

The authors declare that they have no competing interests.

\section{Authors' contributions}

Each of the authors, BA, AA, SKN and WS contributed equally to each part of this work. All authors read and approved the final manuscript. 


\section{Author details}

'Nonlinear Analysis and Applied Mathematics (NAAM)-Research Group, Department of Mathematics, Faculty of Science, King Abdulaziz University, Jeddah, Saudi Arabia. ${ }^{2}$ Department of Mathematics, University of loannina, loannina, Greece.

\section{Publisher's Note}

Springer Nature remains neutral with regard to jurisdictional claims in published maps and institutional affiliations.

Received: 16 January 2019 Accepted: 2 July 2019 Published online: 17 July 2019

\section{References}

1. Kilbas, A.A., Srivastava, H.M., Trujillo, J.J.: Theory and Applications of Fractional Differential Equations. North-Holland Mathematics Studies, vol. 204. Elsevier, Amsterdam (2006)

2. Magin, R.L.: Fractional Calculus in Bioengineering. Begell House Publishers (2006)

3. Klafter, J., Lim, S.C., Metzler, R. (eds.): Fractional Dynamics in Physics. World Scientific, Singapore (2011)

4. Zhou, Y.: Basic Theory of Fractional Differential Equations. World Scientific, Hackensack (2014)

5. Povstenko, Y.Z.: Fractional Thermoelasticity. Springer, New York (2015)

6. Ahmad, B., Alsaedi, A., Ntouyas, S.K., Tariboon, J.: Hadamard-Type Fractional Differential Equations, Inclusions and Inequalities. Springer, Berlin (2017)

7. Petras, I., Magin, R.L.: Simulation of drug uptake in a two compartmental fractional model for a biological system. Commun. Nonlinear Sci. Numer. Simul. 16, 4588-4595 (2011)

8. Ding, Y., Wang, Z., Ye, H.: Optimal control of a fractional-order HIV-immune system with memory. IEEE Trans. Control Syst. Technol. 20, 763-769 (2012)

9. Javidi, M., Ahmad, B.: Dynamic analysis of time fractional order phytoplankton-toxic phytoplankton-zooplankton system. Ecol. Model. 318, 8-18 (2015)

10. Carvalho, A., Pinto, C.M.A.: A delay fractional order model for the co-infection of malaria and HIV/AIDS. Int. J. Dyn. Control 5, 168-186 (2017)

11. Graef, J.R., Kong, L.: Existence of positive solutions to a higher order singular boundary value problem with fractional q-derivatives. Fract. Calc. Appl. Anal. 16, 695-708 (2013)

12. Ahmad, B., Nieto, J.J.: Boundary value problems for a class of sequential integrodifferential equations of fractional order. J. Funct. Spaces Appl. 2013, Article ID 149659 (2013)

13. Zhai, C., Xu, L.: Properties of positive solutions to a class of four-point boundary value problem of Caputo fractional differential equations with a parameter. Commun. Nonlinear Sci. Numer. Simul. 19, 2820-2827 (2014)

14. Henderson, J., Kosmatov, N.: Eigenvalue comparison for fractional boundary value problems with the Caputo derivative. Fract. Calc. Appl. Anal. 17, 872-880 (2014)

15. Li, B., Sun, S., Li, Y., Zhao, P.: Multi-point boundary value problems for a class of Riemann-Liouville fractional differential equations. Adv. Differ. Equ. 2014, 151 (2014)

16. Zhang, L., Ahmad, B., Wang, G.: Successive iterations for positive extremal solutions of nonlinear fractional differential equations on a half line. Bull. Aust. Math. Soc. 91, 116-128 (2015)

17. Ntouyas, S.K., Etemad, S.: On the existence of solutions for fractional differential inclusions with sum and integral boundary conditions. Appl. Math. Comput. 266, 235-243 (2015)

18. Ahmad, B., Ntouyas, S.K., Agarwal, R.P., Alsaedi, A.: Existence results for sequential fractional integro-differential equations with nonlocal multi-point and strip conditions. Bound. Value Probl. 2016, 205 (2016)

19. Zou, Y., He, G.: On the uniqueness of solutions for a class of fractional differential equations. Appl. Math. Lett. 74, 68-73 (2017)

20. Agarwal, R.P., Ahmad, B., Nieto, J.J.: Fractional differential equations with nonlocal (parametric type) anti-periodic boundary conditions. Filomat 31, 1207-1214 (2017)

21. Xu, M., Han, Z.: Positive solutions for integral boundary value problem of two-term fractional differential equations. Bound. Value Probl. 2018, 100 (2018)

22. Ahmad, B., Luca, R.: Existence of solutions for a sequential fractional integro-differential system with coupled integral boundary conditions. Chaos Solitons Fractals 104, 378-388 (2017)

23. Agarwal, R., Hristova, S., O'Regan, D.: Non-instantaneous Impulses in Differential Equations. Springer, Cham (2017)

24. Ahmad, B., Alghanmi, M., Ntouyas, S.K., Alsaedi, A.: Fractional differential equations involving generalized derivative with Stieltjes and fractional integral boundary conditions. Appl. Math. Lett. 84, 111-117 (2018)

25. Ahmad, B., Luca, R.: Existence of solutions for sequential fractional integro-differential equations and inclusions with nonlocal boundary conditions. Appl. Math. Comput. 339, 516-534 (2018)

26. Fernandez, A., Baleanu, D., Fokas, A.: Solving PDEs of fractional order using the unified transform method. Appl. Math. Comput. 339, 738-749 (2018)

27. Mahmudov, N., Emin, S.: Fractional-order boundary value problems with Katugampola fractional integral conditions. Adv. Differ. Equ. 2018, 81 (2018)

28. Ahmad, B., Alsaedi, A., Agarwal, R.P., Alsharif, A.: On sequential fractional integro-differential equations with nonlocal integral boundary conditions. Bull. Malays. Math. Sci. Soc. 41, 1725-1737 (2018)

29. Ahmad, B., Ntouyas, S.K., Alsaedi, A., Shammakh, W., Agarwal, R.P.: Existence theory for fractional differential equations with non-separated type nonlocal multi-point and multi-strip boundary conditions. Adv. Differ. Equ. 2018, Article ID 89 (2018)

30. Su, X.: Boundary value problem for a coupled system of nonlinear fractional differential equations. Appl. Math. Lett. 22, 64-69 (2009)

31. Krasnoselskii, M.A.: Two remarks on the method of successive approximations. Usp. Mat. Nauk 10, $123-127$ (1955)

32. Granas, A., Dugundji, J.: Fixed Point Theory. Springer, New York (2003)

33. Deimling, K.: Multivalued Differential Equations. de Gruyter, Berlin (1992)

34. Lasota, A., Opial, Z:: An application of the Kakutani-Ky Fan theorem in the theory of ordinary differential equations. Bull. Acad. Pol. Sci., Sér. Sci. Math. Astron. Phys. 13, 781-786 (1965) 
35. Covitz, H., Nadler, S.B. Jr.: Multivalued contraction mappings in generalized metric spaces. Isr. J. Math. 8, 5-11 (1970)

36. Kisielewicz, M.: Differential Inclusions and Optimal Control. Kluwer Academic, Dordrecht (1991)

37. Castaing, C., Valadier, M.: Convex Analysis and Measurable Multifunctions. Lecture Notes in Mathematics, vol. 580 Springer, Berlin (1977)

Submit your manuscript to a SpringerOpen ${ }^{\odot}$ journal and benefit from:

- Convenient online submission

- Rigorous peer review

- Open access: articles freely available online

- High visibility within the field

- Retaining the copyright to your article

Submit your next manuscript at $\gg$ springeropen.com 